DEUTSCHES ELEKTRONEN-SYNCHROTRON DESY

DESY $80 / 56$

June 1980

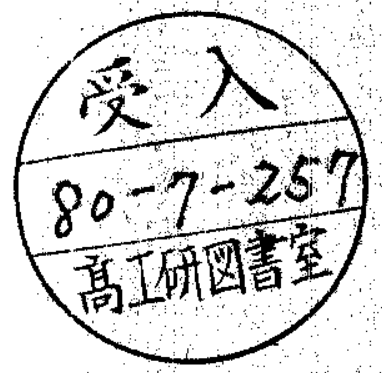

FUTURE ACCELERATOR PLANS AT DESY

by

B. H. Wiik

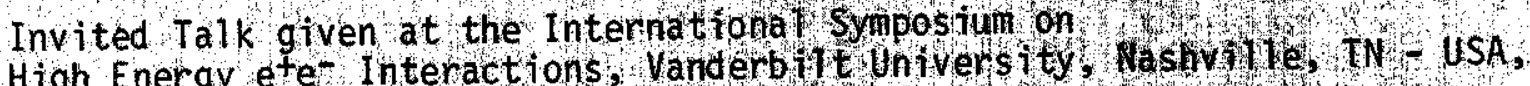
May $1-3,1980$ 
DESY behält sich alle Rechte für den Fall der Schutzrechtserteilung und für die wirtschaftliche Verwertung der in diesem Bericht enthaltenen Informationen vor.

DESY reserves all rights for commercial use of information included in this report, especially in case of apply for or grant of patents.

To be sure that your preprints are promptly included in the HIGH ENERGY PHYSICS INDEX,

send them to the following address (if possible by air mail) :

$$
\begin{aligned}
& \text { DESY } \\
& \text { Bibliothek } \\
& \text { Notkestrasse } 85 \\
& 2 \text { Hamburg } 52 \\
& \text { Germany }
\end{aligned}
$$




\title{
FUTURE ACCELERATOR PLANS AT DESY
}

\author{
B.H.Wiik \\ Deutsches Elektronen-Synchrotron DESY \\ Notkestrasse 85 - 2000 Hamburg 52 - Germany

\section{INTRODUCTION}

Experimental high energy physics has progressed by studying the collisions of what was thought to be the elementary constituents of matter at ever increasing energies. Now leptons and quarks are the basic constituents thus quark-quark, lepton-quark and lepton-lepton collisions are fundamental and should all be investigated. Indeed there is a proliferation of proposals to construct $\mathrm{e}^{+} \mathrm{e}^{-}$facilities to cover the region from PETRA and PEP energies up to and beyond the conjectured mass of the $Z^{0}$. There are three projects, the pp collider at CERN, TEVATRON at FNAL and ISABELLE at BNL aimed at a study of quark-quark collisions. In contrast only DESY has detailed plans 1 to construct a large electron-proton colliding beam facility.

Colliding electrons and protons in a colliding beam machine was first discussed 2 in connection with the ISR but abandoned because of the anticipated low counting rates. After the discovery of the deep inelastic pointlike ep cross section, the interest was revived in 1971 by an LBL/SLAC group ${ }^{3}$. In the meantime several possible electron-proton facilities $4-6$ have been investigated.

At DESY the interest in electron-proton colliding beam machines dates back to 1971-1972. Detailed studies ${ }^{4}$ were then carried out how to use DORIS as a model machine for a larger facility. These plans, abandoned after the $J / \psi$ discovery, were revived in 1978 with the suggestion to install a superconducting magnet ring for protons in the PETRA tunnel With this option, called PROPER, it would be possible to collide $17.5 \mathrm{GeV}$ electrons with $280 \mathrm{GeV}$ protons in eight intersection regions.

The physics potential 7 of large electron-proton colliding rings was discussed in a meeting 8 organized jointly by DESY and ECFA in Hamburg in March of 1979. The study of neutral and charged weak currents is one of the prime motivations for constructing a large electron-proton colliding beam facility. Indeed the properties of charged currents at small distances can only be explored using colliding electron proton machines. It is crucial that a new accelerator is able to explore the region above $100 \mathrm{GeV}$ in the center of mass system, the presumed mass scale of weak interactions, where new phenomena might be expected to occur. Although the kinematical region liberated by PROPER would greatly exceed that available at present fixed target machines the meeting concluded that the energy was to low to explore the region above $100 \mathrm{GeV}$ with sufficient rate. DESY therefore investigated various possibilities aimed at raising the energy and found that to install a ep machine in a $6.5 \mathrm{~km}$ long tunnel ajoining the DESY site is the favoured option. This machine called HERA (Hadron-Elektron-Ring-Anlage) is designed to collide $820 \mathrm{GeV}$ protons with $30 \mathrm{GeV}$ electrons yielding $314 \mathrm{GeV}$ in the center 
of mass and a maximum momentum transfer squared of $98400 \mathrm{GeV}^{2}$. This is equivalent to an electron of $52000 \mathrm{GeV}$ impinging on a proton at rest HERA thus explores a similar kinematical range as that accessible to LEP and various $p p$ ( $p \bar{p}$ ) colliders. In the interesting region above $10^{4} \mathrm{GeV}^{2}$ HERA produces roughly 100 times more events than PROPER 5 for the same luminosity.

A feasibility study ${ }^{1}$ of this project done in collaboration with ECFA has been completed. The project has received strong support from the German high energy community and a project definition endorsed by ECFA has been forwarded to the German Government. A first response is expected later this year and this might be followed by a decision in 1981.

A programme to develop superconducting magnets suitable for HERA is underway and the first prototypes are expected to be available in 1982. The construction time of the electron ring is estimated to be five years compared to some seven years for the proton ring. Since superconducting magnets presumably take longer to produce and test than to install, some of this time can be used to study $\mathrm{e}^{+} \mathrm{e}^{-}$ collision between $40 \mathrm{GeV}$ and $70 \mathrm{GeV}$ in the center of mass using the HERA electron ring. Originally DESY has proposed 8 to upgrade PETRA to cover this energy range and a relatively modest improvement programme is now underway aimed at reaching about $45 \mathrm{GeV}$ in the center of mass. Since superconducting RF cavities or a pulsed RF system are needed to extend this to higher energies it seems advantagous to use the larger radius electron ring of HERA to cover the energy region up to $70 \mathrm{GeV}$ with a conventional RF system. Indeed this can be done at little extra cost with the RF system as foreseen for the ep option.

In this talk I'll describe the HERA project, first the physics and then the machine. The talk is based on the ECFA-DESY report listed in reference 1. A more complete list of references can be found in that report.

\section{THE PHYSICS PROGRAMME AT HERA}

Electron-Proton Collisions

It has been found experimentally that for large values of $Q^{2}$ the incident electron interact directly with one of the quarks in the nucleon as shown in Fig. 1. The interaction is. mediated by spacelike currents charged or neutral and is characterized by the kinematical quantities $Q^{2}, v$ or the scaling variables $x$ and $y$ defined in Fig. 1.

A deep inelastic event has a rather striking final state topology which makes it easy to recognize among the much more numerous beam gas events. As indicated in Fig. 1 the scattered lepton appears at a large angle with respect to the beam axis with its transverse momentum balanced by the struck quark which fragments into a jet of hadron appearing on the opposite side of the beam axis. The remains of the proton give rise to a forward jet of hadrons with no net transverse momentum with respect to the beam axis. Because of the imbalance between the incident electron and proton momenta the particles will in general emerge in the forward hemisphere along the 


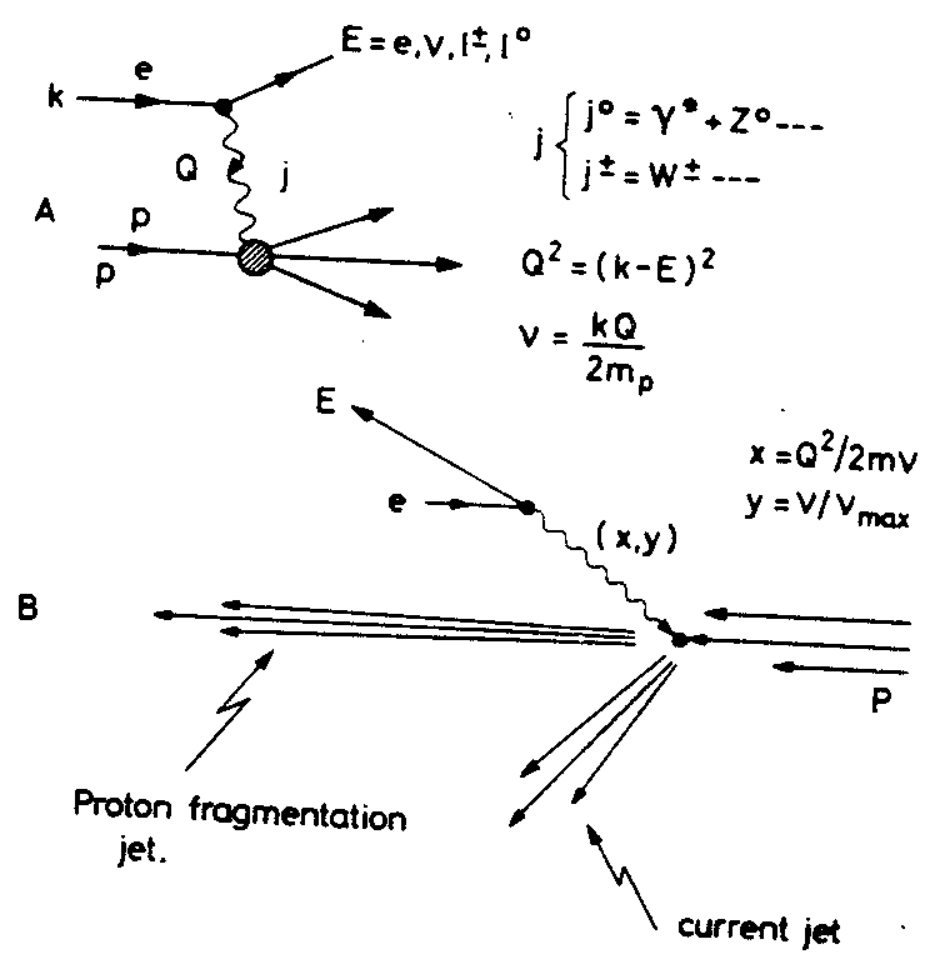

C

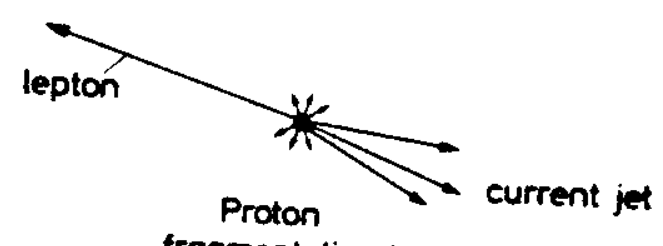

frogmentation jet

Fig. 1 - Kinematics of deep inelastic electron-proton collisions

proton direction. The proton jet, the quark jet and the lepton defines a plane with small momenta transverse to the plane and large momenta in the plane.

The Monte Carlo simulation of a deep inelastic charged current event is shown in Fig. 2, where the transverse and longitudinal momentum of each particle is plotted with respect to the beam axis. In this event the struck quark radiates a gluon leading to three well separated hadron jets, the outgoing $v$ is not shown. Because of their distinctive signature it will presumably be possible to identify deep inelastic events cleanly. Very low counting rate experi- 
ment: are therefore feasibie.

$$
\begin{aligned}
& q^{2}=25000 \mathrm{GeV}^{2} \\
& x=0.5
\end{aligned}
$$

Gevle

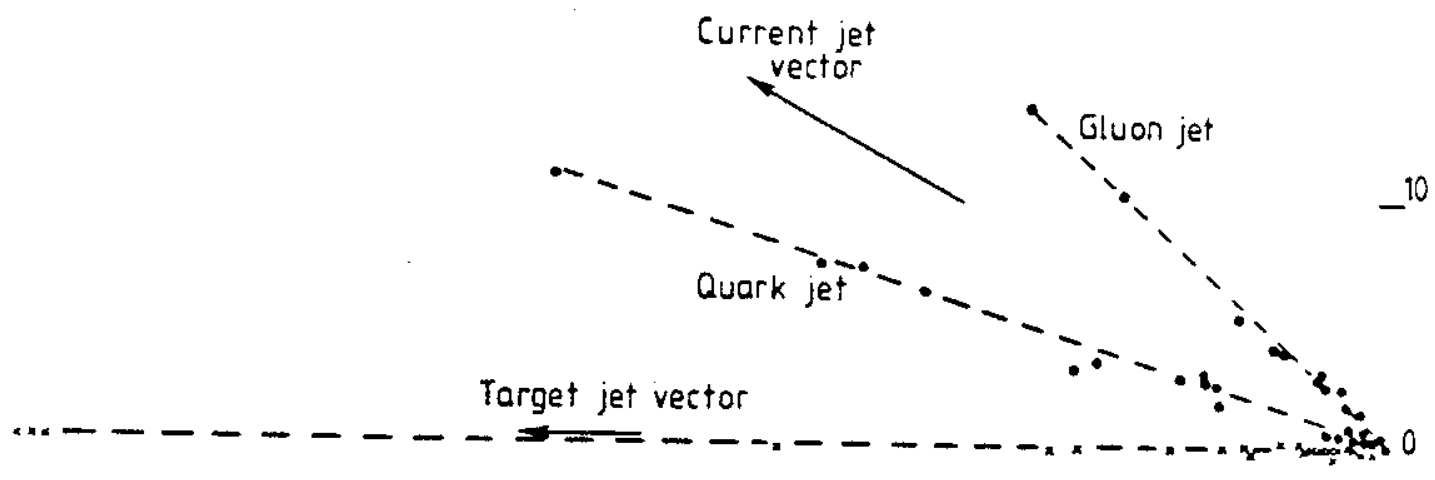

$\begin{array}{cccccc}\text { GeVic } & 1 & 1 & 1 & 1 & 1 \\ 0 & 40 & 30 & 20 & 10 & 0\end{array}$

Fig. 2 - Simulated ep $\rightarrow$ ux event. Plotted are longitudinal and transverse moments of the produced hadrons with respect to the proton direction. Note that the struck quark radiates a gluon

The kinematical region available with HERA is shown in Fig. 3. In the bottom left corner the region covered by a $1 \mathrm{TeV}$ fixed target machine is shown. It is clear that HERA opens up a totally new kinematical range. Furthermore it does not seem profitably to extract the proton beam for conventional fixed target experiments in particular since such a venture would double the cost of the proton part of the project.

The $Q^{2}$ value at which the electromagnetic and the weak interaction is expected to coalesce ${ }^{9}$ is shown as the horizontal dotted line. A large kinematical area is available above this line and we will show below that the rate is sufficient to explore a part of this region.

A deep inelastic process is well defined and the data can be used to determine the properties of these currents - charged or neutral - at very smal1 distances. Conversely once the currents are understood, measurements can explore the protons and its constituents down to distances of $10^{-17} \mathrm{~cm}$. The spectrum of electronlike leptons or the spectrum of heavy quarks which couple to $u$ or d quarks can be investigated up to a mass of $200 \mathrm{GeV}$ with HERA. 


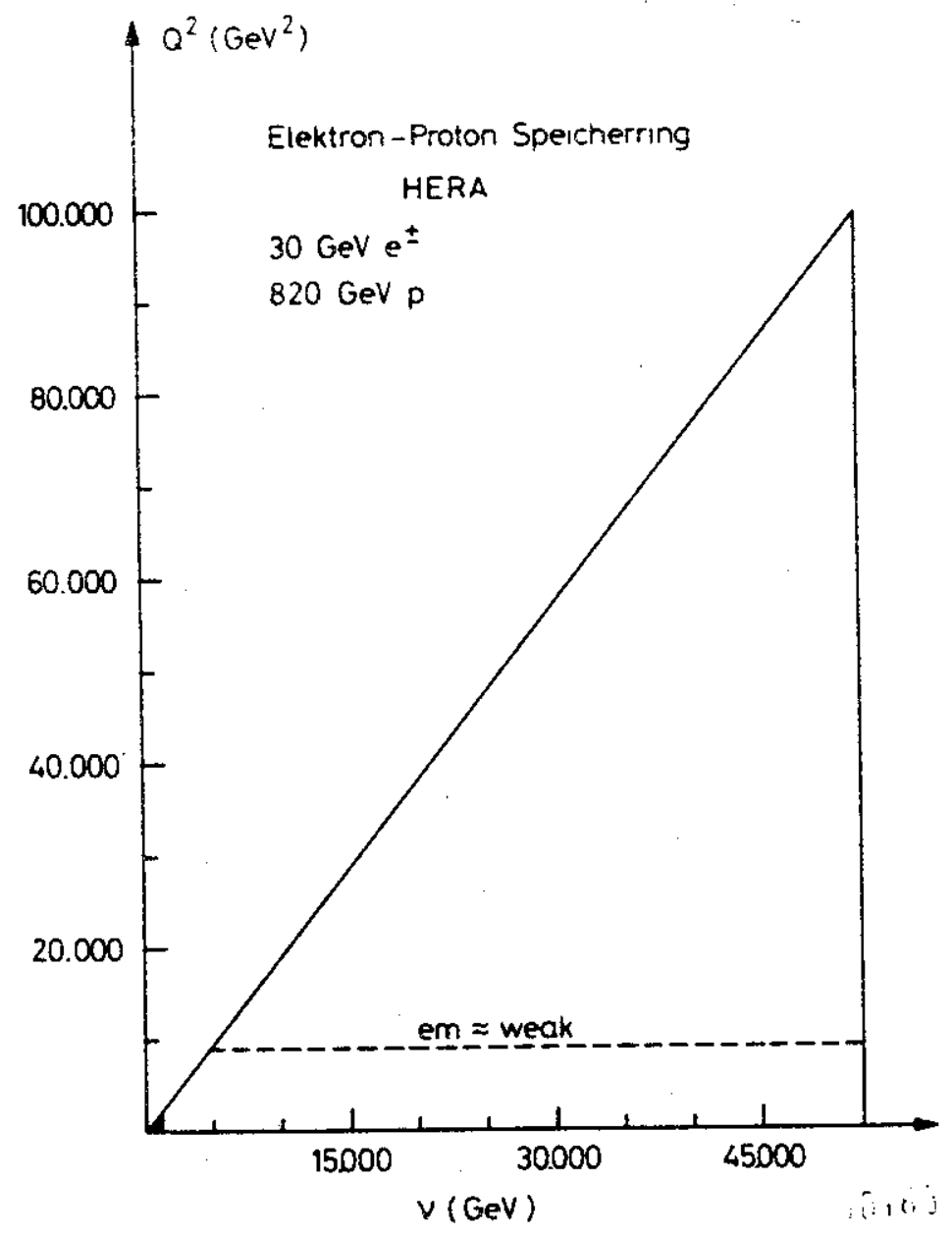

Fig. 3

Kinematical region in $Q^{2}$ and $v$ which can be explored with HERA

$Q^{2}$ It is important to bear in mind that for small values of $Q^{2}$ is equin the photons are nearly real and hence the electron beam is equivalent to a well defined bremsstrahlungs beam with an end point energy of $52000 \mathrm{GeV}$. Therefore also classical hadron physics can be investigated with HERA up to very large energies.

Charged current events

To observe the charged weak current at small distances is one of the prime motivations for the construction of a large electronproton colliding beam facility. Present data are all consistent with a lefthanded current which is mediated by a single charged vector boson with a mass around $80 \mathrm{GeV}$. However, present experiments can only probe the weak interaction at $Q$ values which are small compared to the characteristic mass scale of the weak interactions. The observed simplicity of the charged current might reflect only the static limit studied so far and a rich structure with many vector bosons, some perhaps giving rise to righhanded currents, might appear at higher energies. Measurements at HERA will enable us to investigate the region well beyond $80 \mathrm{GeV}$ and answer these questions. 
From a purely experimental point HERA has some unique features compared to present fixed target experiments.

- Very high energy.

The beam is equivalent of a monoenergetic neutrino beam with an energy up to $52 \mathrm{TeV}$

- Choice of helicity.

It will presumably be possible to change the helicity of the incident lepton - i.e. the cross section for left and righthanded electrons (or positrons) can be measured directly.

- Visibility.

The target is massless and can be surrounded by fine grained detectors including particle identification.

- Favourable kinematics.

The lepton, the current jet and the target fragmentation jet are presumably well separated in space and the event is easily recognized.

The number of charged current events expected in a bin $d x d y=(0.2)^{2}$ after one month of data taking with an unpolarized $30 \mathrm{GeV}$ electron beam colliding with protons of either $820 \mathrm{GeV}$ or $200 \mathrm{GeV}$ is shown in Fig. 4, assuming a luminosity of $10^{32} \mathrm{~cm}^{-2} \mathrm{~s}^{-1}$ The rates were estimated in the standard model 9 with $m_{w}=78 \mathrm{GeV}$ and formfactors parametrized according to Buras and Gaemers 10 .

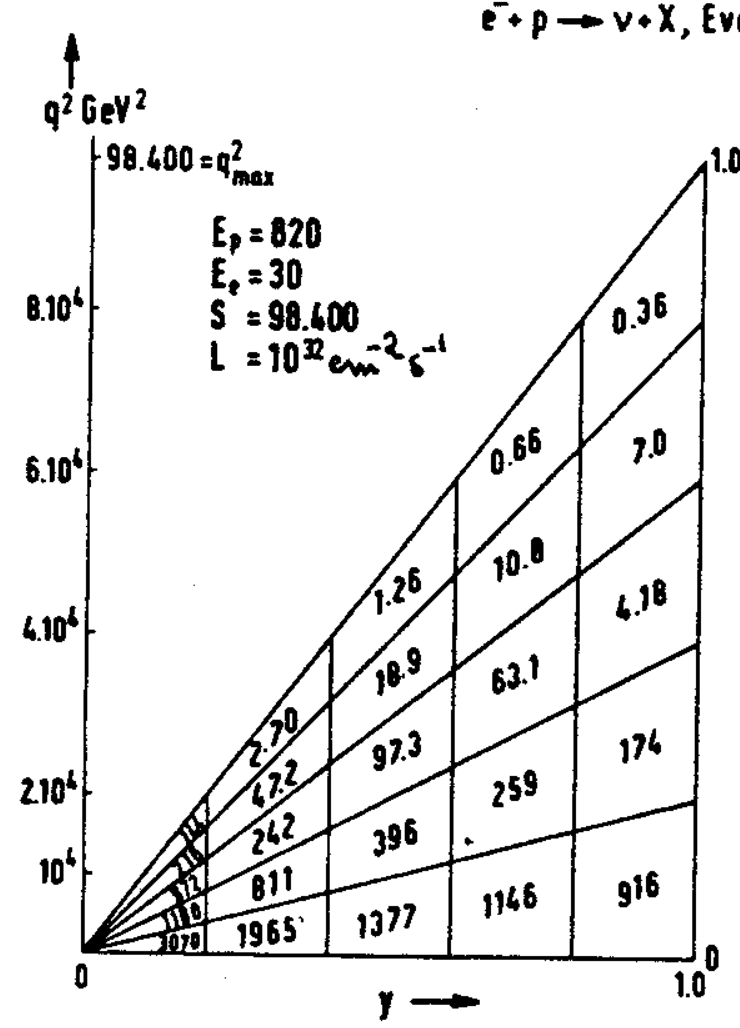

$$
e^{-}+p \rightarrow v+x \text {, Events per month (unpolarized electrons) } 3016 \hat{y}
$$

$q^{4} \operatorname{cev}^{2}$

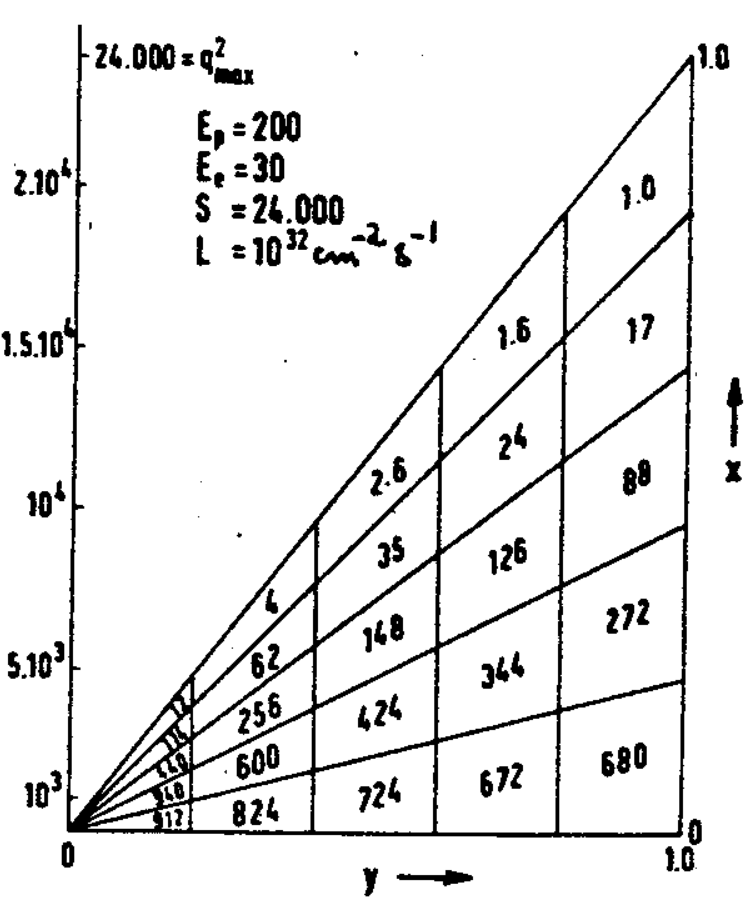

Fig. 4 - Number of charged current events produced per month of running time. In bins $d x d y=(0.2)^{2}$ assuming the standard model 
Given the distinctive signature of a charged current event $j t$ seems possible to measure the cross section for $Q^{2} \leq 4 \times 10^{4} \mathrm{GeV}^{2}$. The expected counting rate for $e^{-p} \rightarrow \nu x$, evaluated with the assumptions 1 isted above, is plotted in Fig. 5 versus $Q^{2}$ for various propagator masses. It is clear that HERA experiments can be used to determine the mass of the propagator as long as it is below say $300-400 \mathrm{GeV}$. The data can also be used to determine whether the charged current is dampted by a single vector boson as presently believed or by several. As an example, a model containing two vector bosons has been evaluated with the assumption that they have the same coupling constant at $Q^{2}=0$ and that one of the vector bosons has a mass of $78 \mathrm{GeV}$. The expected event rate for the two vector boson model, normal ized to the standard model event rate, is plotted in Fig. 6 versus $Q^{2}$ for various mass values of the second vector boson. The effects are large.

The existance of righthanded currents can of course be deduced directly from a measurement of $\sigma\left(e_{R}^{-} p \rightarrow v x\right)$ or $\sigma\left(e_{L}^{+} p \rightarrow \bar{v} x\right)$.

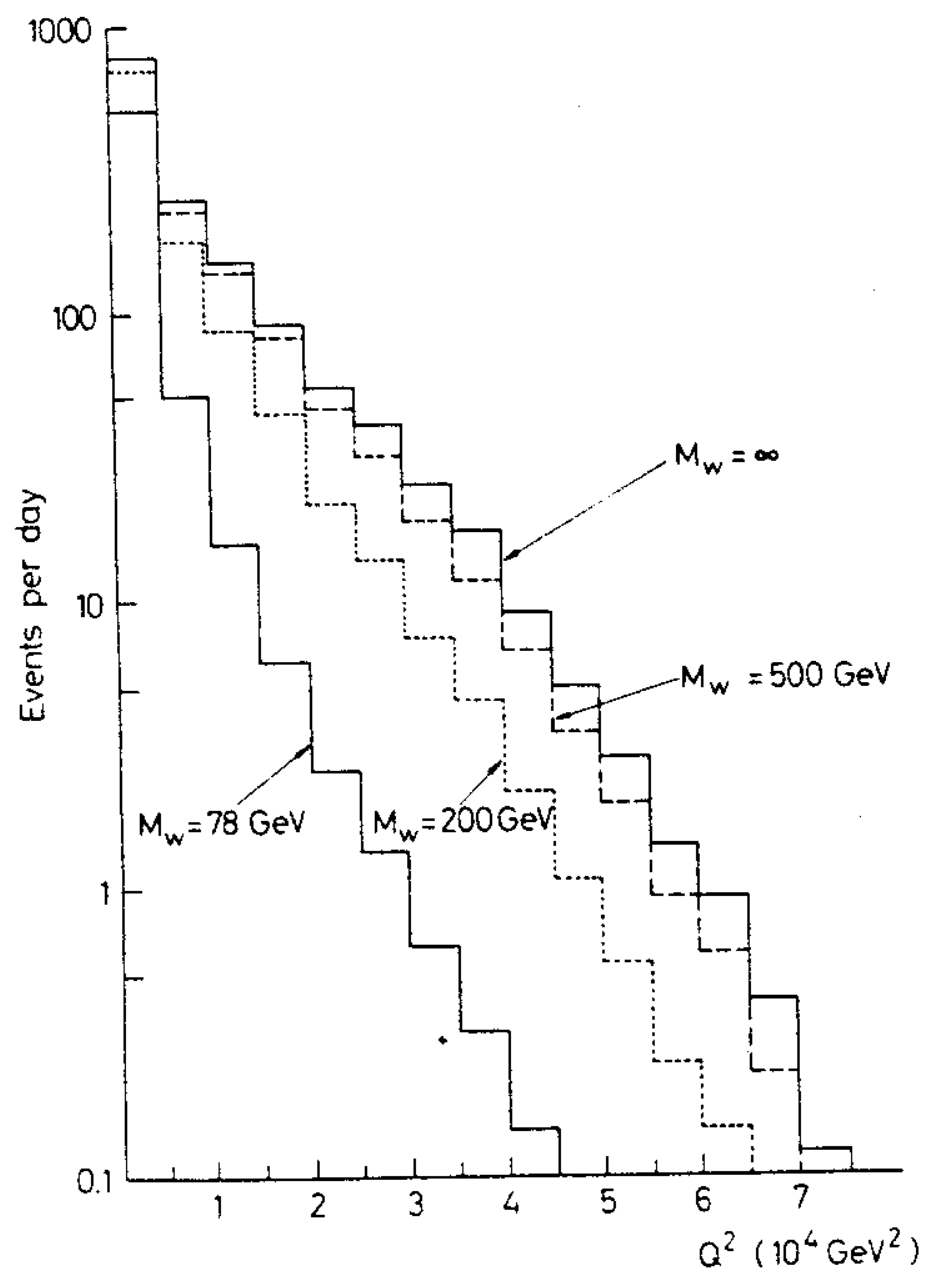

Fig. 5

Events per day for

$e^{-}+p \rightarrow v+x$

in $Q^{2}$ bins of

$5000 \mathrm{GeV}^{2}$ with the

standard as sump-

tions 


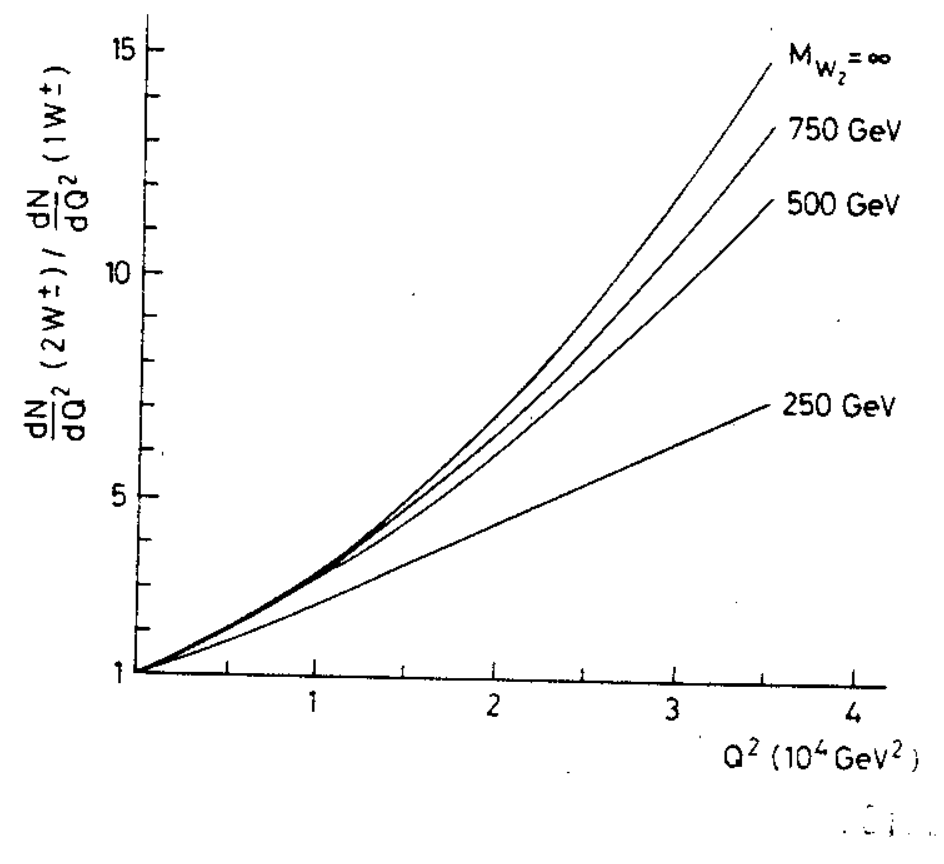

Fig. 6 - Ratio $d N / d Q^{2}\left(\right.$ two $\left.W^{ \pm}\right) / d N / d Q^{2}$ (one $W^{ \pm}$) for
different mass values of the second $W^{ \pm}$ Experimental remarks

The obvious experimental questions are:

1) Can charged current events ep $\rightarrow v x$ be separated from the neutral currept events ep $\rightarrow$ ex ?

2) Can $Q^{2}$ and $\nu$ be measured with sufficient precision for each event and can the formfactors be extracted from these data? The answer to both questions is: yes - provided the measurements are done using a large solid angle detector without holes in the acceptance. An example of such a detector is shown in Fig. 7.

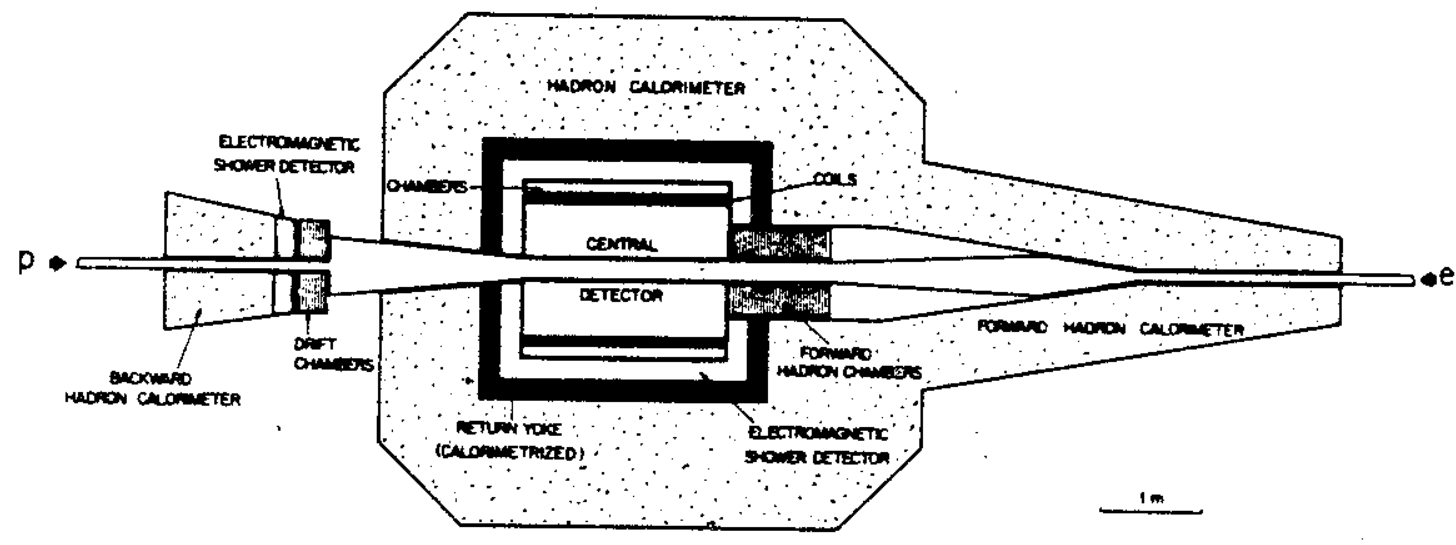

S.?.

Fig. 7 - Schematic layout of a detector optimized for charged current events ep $\rightarrow$ vx 
Interesting deep inelastic events can be separated from beamgas events by summing the transverse momenta of the final state hadrons with respect to the beam axis and demanding that this sum is above a few GeV. The direction of the final state lepton can be roughly determined from a measurement of the final state hadrons. One then only needs to examine the detector if it registered a single high energy electron travelling within the solid angle defined by the hadrons or nothing. Since weak and the electromagnetic cross section are expected to be similar this should lead to a rather complete separation.

The resolution expected in $Q^{2}$ and $x=Q^{2} / 2 m v$ from a measurement of the hadron final state only using the calorimeter type detector depicted in Fig. 7 is shown in Fig. 8. The detector measures angles and momenta with the uncertainties listed in table 1.

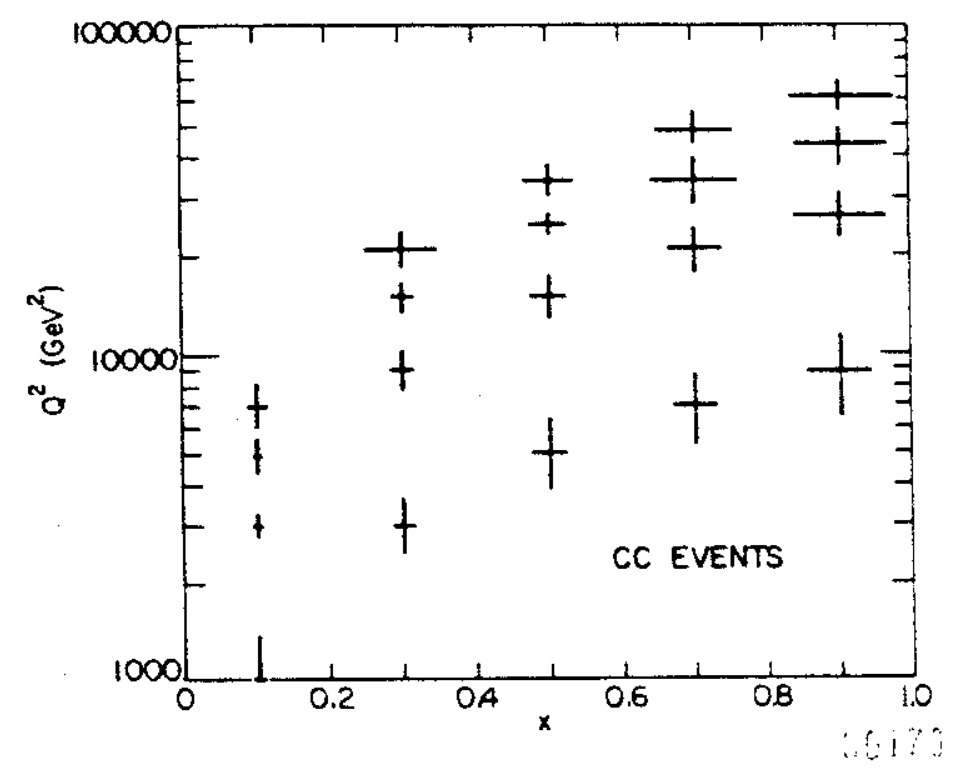

Fig. 8

Measurement errors in $\mathrm{Q}^{2}$ and $\mathrm{x}$ for the resolution listed in table 1

Table 1 - Detector resolution

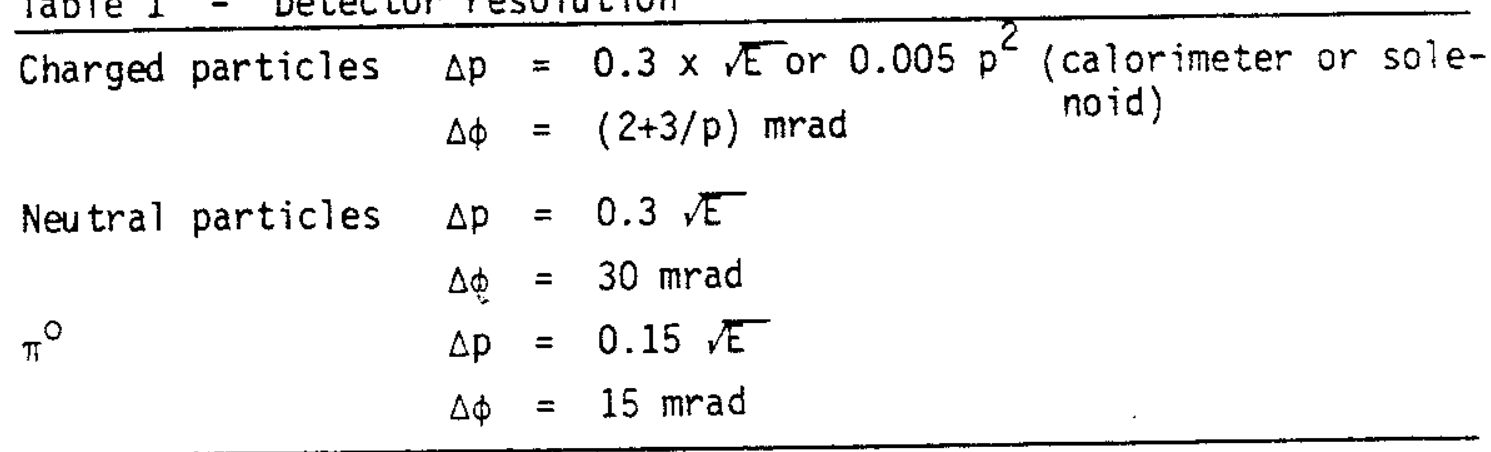


Furthermore also the formfactors can be determined with a good precision using such a detector. This is shown in Fig. 9, where the ratio of the measured formfactor to the input formfactor is plotted versus $Q^{2}$ for different values of $x$. For this computation it was assumed that all particles travelling within 30 mrad of the proton beam or within 45 mrad of the electron beam are lost. It is clear that the $Q^{2}$ region between a few hundred $\mathrm{GeV}^{2}$ up to $40000 \mathrm{GeV}^{2}$ can be covered with good precision for proton energies between $820 \mathrm{GeV}$ and $200 \mathrm{GeV}$ and an electron energy of $30 \mathrm{GeV}$.

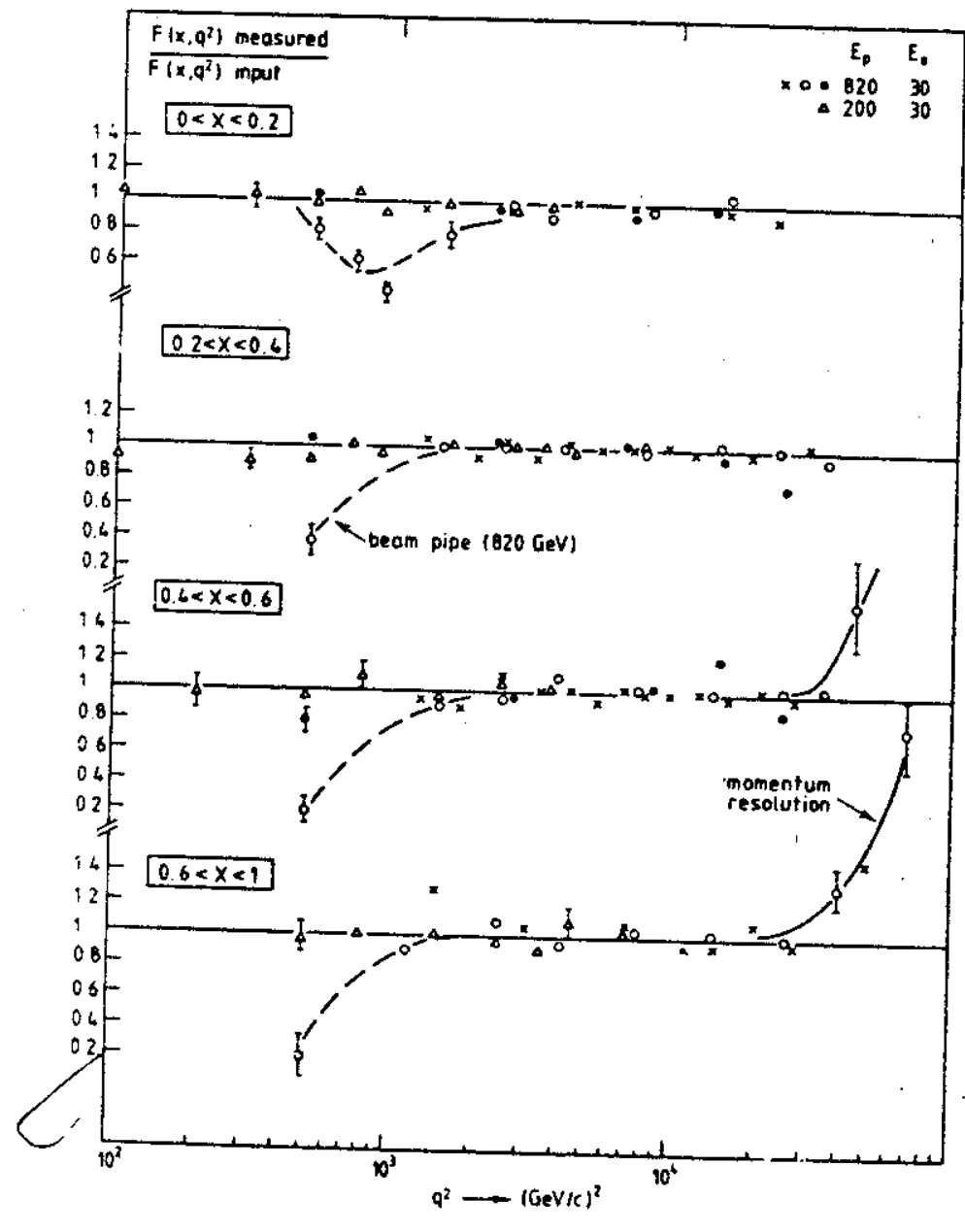

Fig. 9

Ratio of reconstructed to input structure functions for charged current events generated by three independent Monte Carlo programs

'New Fermions

HERA is ideally suited to produce electronlike charged or neutral leptons and new heavy quarks which couple to $u$ or d quarks in the proton. We know that such couplings are rather weak in the standard model, however, new currents may exist. Indeed the basic 
fermions must have excited states if they are not pointlike. The rate for producing a heavy quark from a light quark is plotted in Fig. 10 with the mass of the lepton at the upper vertex (Fig. 1) as a parameter. The rates were evaluated with the assumptions 1 isted above plus the assumption that the new current couples with the same strength as the old one. Leptons and quarks with masses up to 150 - $200 \mathrm{GeV}$ can be found in this way. The decay of these particles lead to rather spectacular signatures as shown in Fig. 11 for ep $\rightarrow L^{0} \times$ with $L^{0} \rightarrow e^{-} Q q^{\prime}$.

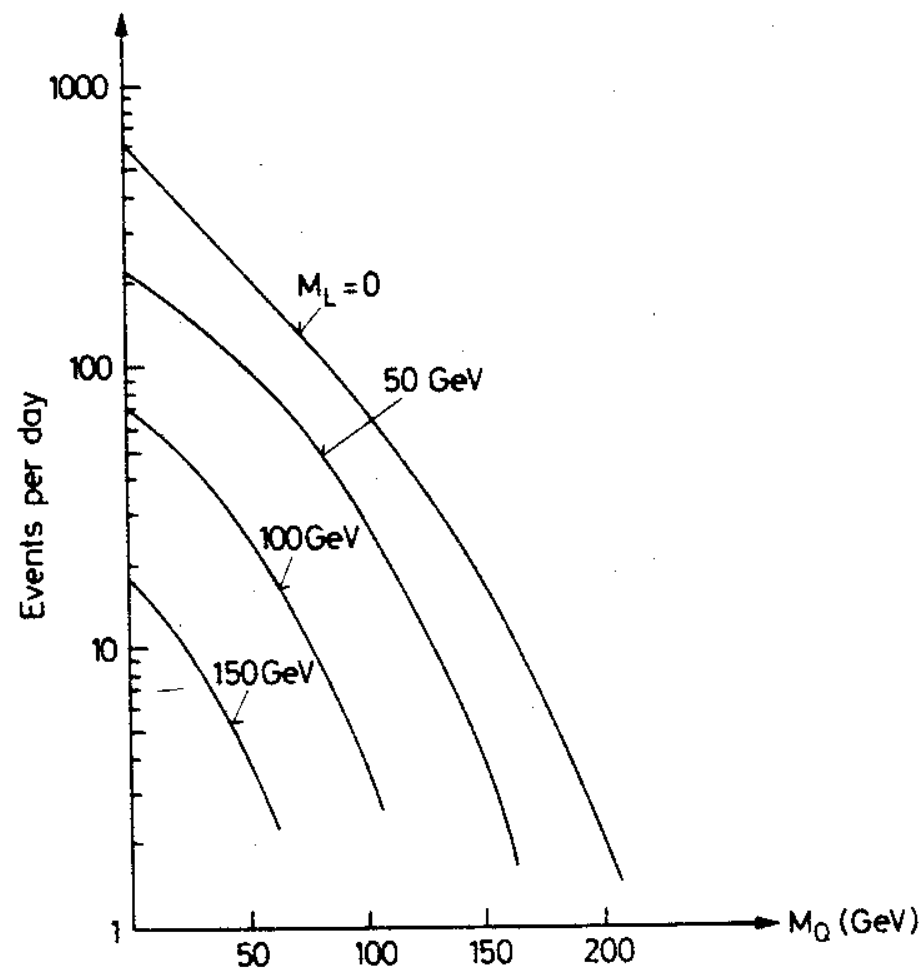

Fig. 10

Number of events per day for

$e^{-}+p \rightarrow L^{0}+Q+x$

at $s=9.6 \times 10^{4} \mathrm{GeV}^{2}$ assuming lefthanded couplings, unpolarized electrons, $m_{w^{\prime}}=78 \mathrm{GeV}$, Buras-Gaemers QCD parametrization with $\Lambda=0.5 \mathrm{GeV}$ and a luminosity of $10^{32} \mathrm{~cm}^{-2} \mathrm{~s}^{-1}$ 


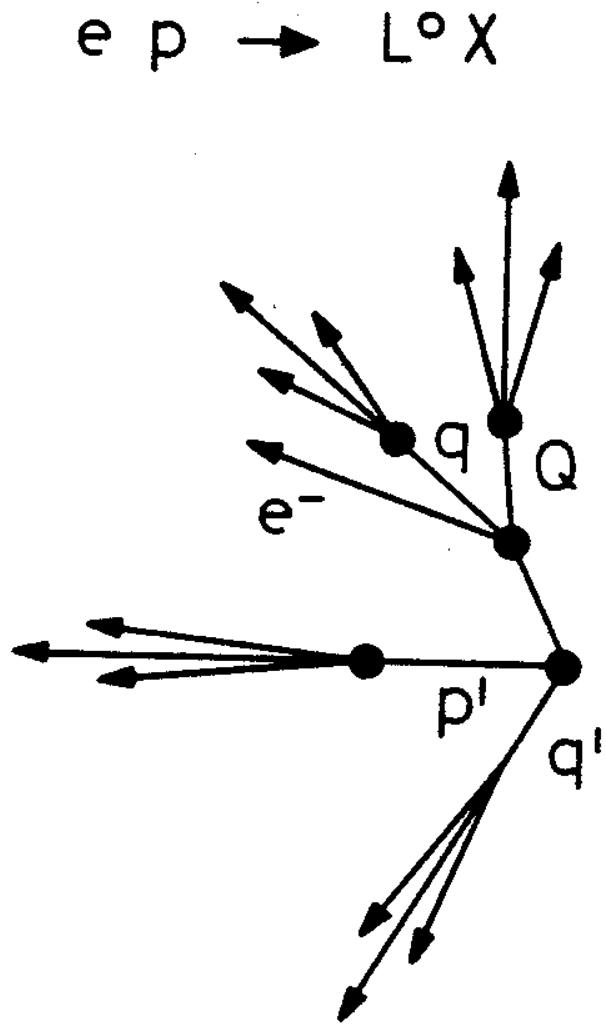

Fig. 11

Topology of

$\mathrm{e} p \rightarrow \mathrm{L}^{0} \mathrm{x}$ with

$L^{0} \rightarrow e^{-} q Q$

Neutral Currents

One photon exchange and $Z^{0}$ exchange contribute (Fig. 1) coherently to $e+p \rightarrow e^{\prime}+x$ and both contributions are of similar strength at HERA energies. Measurements of this process can therefore decide if indeed the electromagnetic and the weak interactions are manifestations of a single force and if this unification occurs as conjectured in the Salam-Weinberg model ${ }^{9}$ or if a more complicated mechanismn involving many $Z^{\circ} \mathrm{s}$ is realized in nature. The number of neutral current events produced in a bin $\mathrm{d} x \mathrm{dy}=(0.2)^{2}$ per day by $30 \mathrm{GeV}$ electrons colliding with $820 \mathrm{GeV}$ protons and a luminosity of $10^{32} \mathrm{~cm}^{-2} \mathrm{~s}-1$ is plotted in Fig. 12 . Again due to the characteristic topology of deep inelastic events HERA can extend the $Q_{2}^{2}$ range from the present few hundred $\mathrm{GeV}^{2}$
out to some $30000 \mathrm{GeV}^{2}$. 


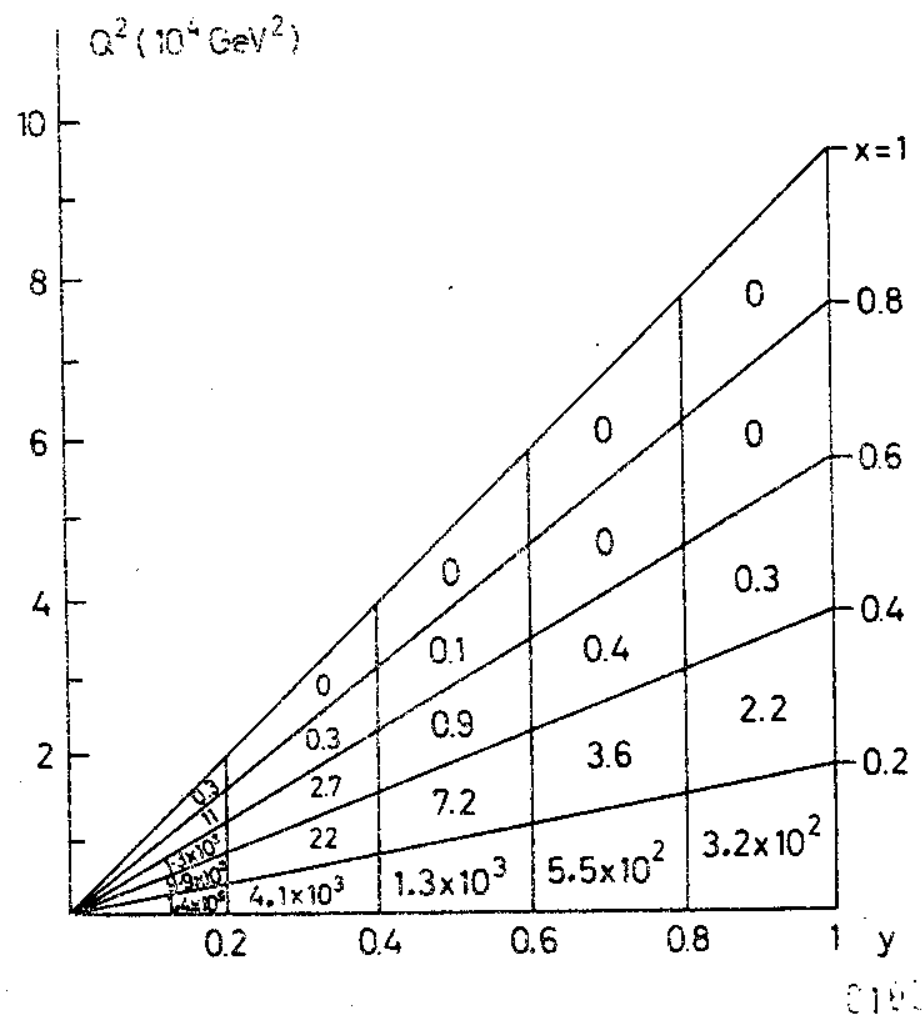

Fig. 12

Number of events per day for $\mathrm{e}^{-}+\mathrm{p} \mathrm{e}^{-}+\mathrm{x}$ at $s=9.6 \times 10^{4} \mathrm{GeV}^{2}$ and the standard assumptions

The presence of a weak current in the amplitude has clear signatures.

1) Parity viclation

$$
\begin{aligned}
& \sigma\left(e_{L}^{-} p+e^{-\cdot} x\right) \neq \sigma\left(e_{R}^{-} p+e^{-} \cdot x\right) \\
& \sigma\left(e_{L}^{+} p+e^{+} x\right) \neq \sigma\left(e_{R}^{+} p+e^{+} x\right)
\end{aligned}
$$

This effect can on 3 be caused by a neutral weak current.

2) Appearant C-violation

$$
\begin{aligned}
& o\left(e_{L}^{-} p \rightarrow e^{-,} x\right) \neq \sigma\left(e_{L}^{+} p \rightarrow e^{+,} x\right) \\
& \sigma\left(e_{R}^{-} p \rightarrow d^{-}, x\right) \neq \sigma\left(e_{R}^{+} p \rightarrow e^{+} x\right)
\end{aligned}
$$

Two-photon exchange wili also give rise to a charge asymetry. This effect, however, is expected to be of order $\alpha / \pi \ln Q^{2} / \mathrm{m}^{2}$ with $m \sim 300 \mathrm{MeV}$. At large values of $Q^{2}$ this effect is small compared to the charge asympetry caused by $Z^{0}$ exchange and it has furthermore a different $Q^{2}$ dependence. The two photon effects can be determined at relatively low values of $Q^{2}$ where $Z^{\circ}$ exchange has a small effect only. 
3) The presence of a $1-(1-y)^{2}$ term which is not allowed in the one photon exchange approximation. This effect cannot be caused by
two photon exchange.

The size of these effects in the standard model is shown in Fig. 13 where the ratio for the left and righthanded electrons and positrons is plotted as solid 7 ine versus $y$ for $x=0.25$ and $s=9.8 \times 10^{4} \mathrm{GeV}^{2}$. Note that the rates are sufficient to determine these asymmetries in a few months of running.
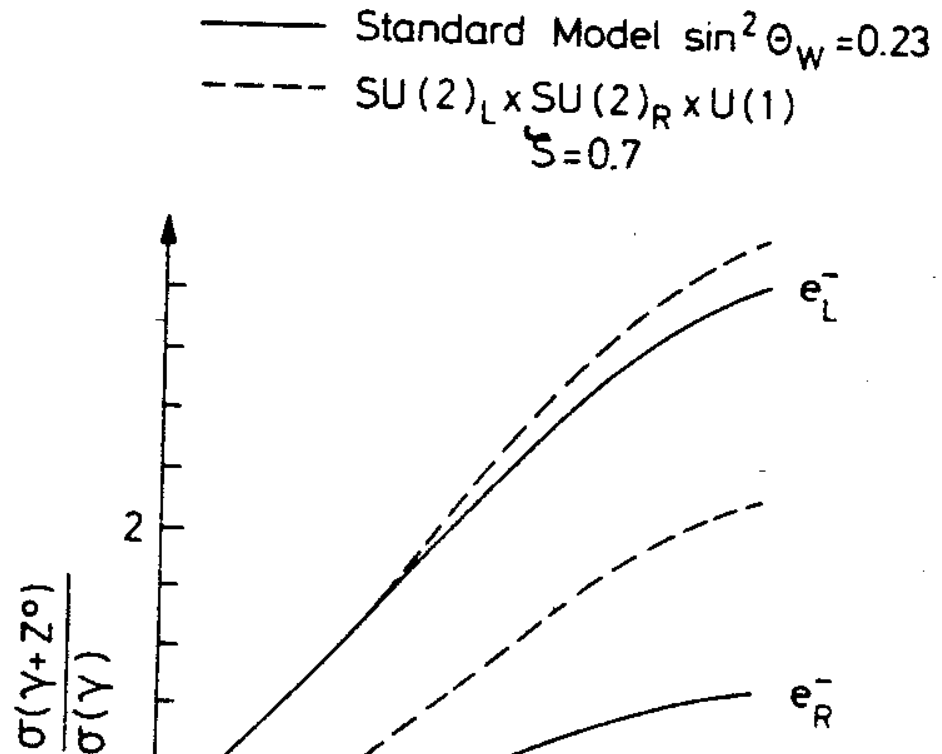

Fig. 13

The ratio

$\sigma\left(\gamma+z^{0}\right) / \sigma(\gamma)$

at $x=0.25$ and

$s=9.6 \times 10^{4} \mathrm{GeV}^{2}$

for two different weak interaction models.

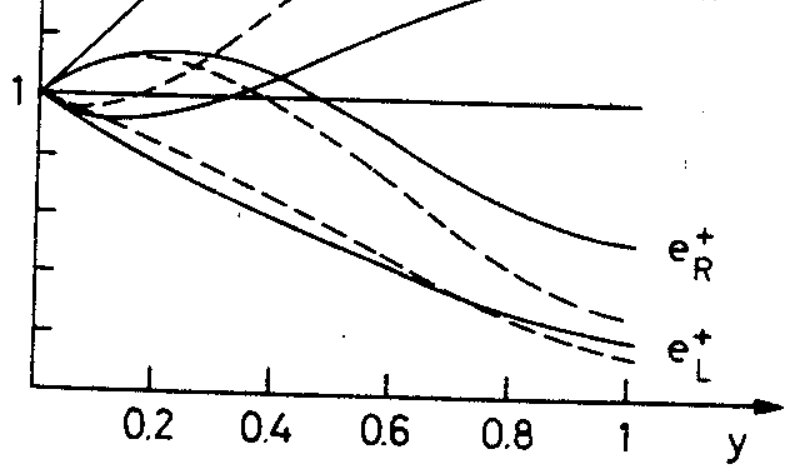

.30103

A measurement of these asymmetries can be used to pin down the properties of the neutral weak current. Suppose that $S U(2)_{L} x$ $S U_{R}(2) \times U(1)$ is realized in nature. The parameters of ${ }_{L}$ such a group can be adjusted to agree with all known data. The dotted lines in Fig. 13 show the cross sections expected in this model with the mass of the second $Z^{0}$ at $224 \mathrm{GeV}$. It is clear that the two models can be separated.

New flavour changing neutral currents might also appear. Such currents could lead to spectacular processes like $e^{-d} \rightarrow \tau^{-} b$. 
Test of Strong Interactions

QCD 11 makes clear, unambigous predictions for deep inelastic processes.

Such a prediction for non-singlet moments is plotted in Fig. 14 versus $Q^{2}$. Note that the value of the moments in $Q C D$ is nearly con-
stant for $Q^{2}$ above $1000 \mathrm{GeV}^{2}$. This is a very strong prediction unique to QCD. For example a simple power behaviour expected in other types of field theories can mimic the observed behaviour over the present available $Q^{2}$ range - however it will deviate from the QCD predictions at large values of $Q^{2}$. This constancy makes it also easy to observe threshold like color liberation if it should occur. Measurements of the final state hadrons will enable us to carry out detailed tests of QCD.

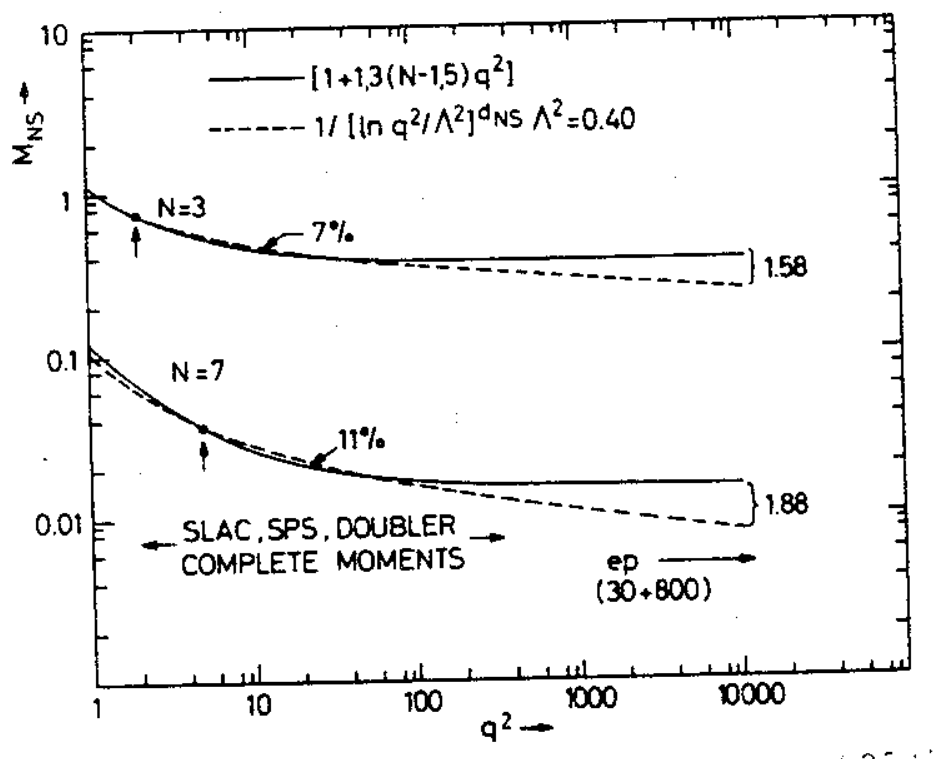

Fig. 14

A simple $1 / 0^{2}$ power behavior compared with the QCD logarithmic behavior for non-singlet moments

Probing Quarks and Leptons

Faced with the large numbers of leptons and quarks many physicists find it natural that these entities are made up of new building blocks. With HERA we can probe the fermion structure down to distances of $10^{-17} \mathrm{~cm}$ corresponding to $10^{-4}$ of the size of the proton.

If the leptons have a size we would expect to observe a leptonic form factor and ultimately the production of excited leptons. The cross section would be modified by a form factor $F\left(Q^{2}\right)=1 /\left(1+q^{2} / M^{2}\right)$ giving rise to a scaling violation which is very djfferent from that expected in QCD. A $10 \%$ measurement at $4 \times 10^{4} \mathrm{GeV}^{2}$ would be sensitive to any mass of the order of $1 \mathrm{TeV}$. An excited lepton could decay into $e+\gamma, e+z^{0}$ and $e+W$ leading to peaks in the invariant spectrum. 
The cross section would also be modified in the case of a quark structure in a similar manner - j.e. again one might probe down to distances of $(1 \mathrm{TeV})^{-1}$. Aga in there might be excited quark states. Another possibility is that the proton contains new gluon-like particles which interact neither weakly nor electromagneticaliy. These particles would show as a step in the momentum fraction of the protons carried by the quarks. Example of such particles are the spin 1/2 gluinos expected in supersymetric theories. The expected behaviour of the momentum 12 sum rule in crossing the threshold $Q^{2}=4 \mathrm{~m}_{\mathrm{g}}^{2}$ is shown in Fig. 15 .

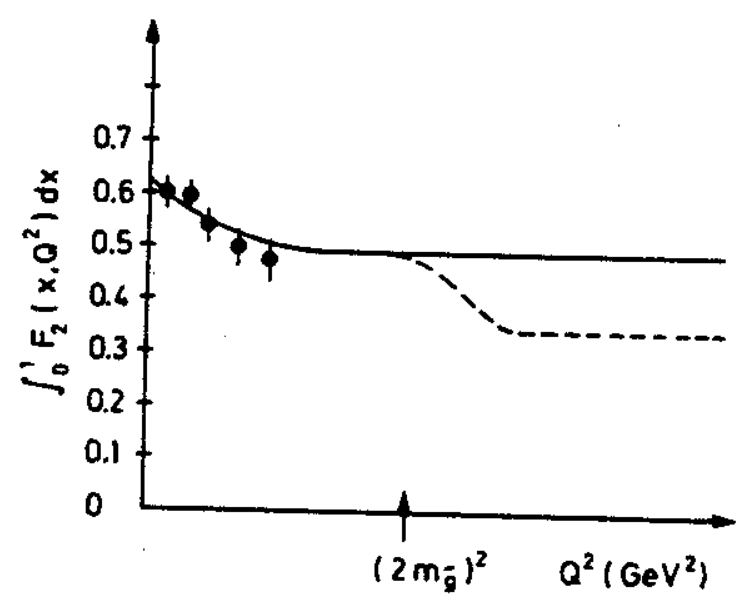

Fig. 15

Possible behavior of the momentum sum rule as the threshold $\mathrm{Q}^{2}=$ $4 m_{9}^{2}$ is crossed

$\mathrm{e}^{+} \mathrm{e}^{-}$Physics below the $z^{0}$

The $\mathrm{e}^{t} \mathrm{e}^{-}$annihilation cross section evaluated in the standard model with 3 generations and $\sin ^{2} \theta_{w}=0.25$, is plotted in Fig. 16 versus energy. The energy range covered by HERA is indicated. At the upper end of that range $R=\sigma\left(\mathrm{e}^{+} \mathrm{e}^{-} \rightarrow\right.$ hadrons $) / \sigma\left(\mathrm{e}^{+} \mathrm{e}^{-} \rightarrow \mu^{+} \mu^{-}\right)$ is about 30 compared to an $R$ value of maybe 5 for purely one photon annihilation indicated by the dotted line. The cross section is thus dominated by the $Z^{0}$-pole and the same decays as on the $Z^{\circ}$ peak can be studied except for a $20 \mathrm{GeV}$ difference in mass. About $3000 Z^{0}$ decays per day are observed with a luminosity of $10^{32} \mathrm{~cm}^{-2} \mathrm{~s}^{-1}$. The region covered by HERA is also well suited to determine the coupling constants of the neutral current to the various fermions. In Fig. ' 17 the forward backward asymmetry expected in the standard model for various types of fermions is plotted versus center of mass energy squared in units of the $Z^{\circ}$ mass squared. Essentially, this asymmetry measures the product of the axial couplings of the electron and the outgoing fermions to the neutral current. By colliding an unpolarized beam with a polarized beam one can measure the product of the vector and the axial couplings. It seems 
feasible to produce polarization at HERA whereas it seems to be more difficult at LEP.

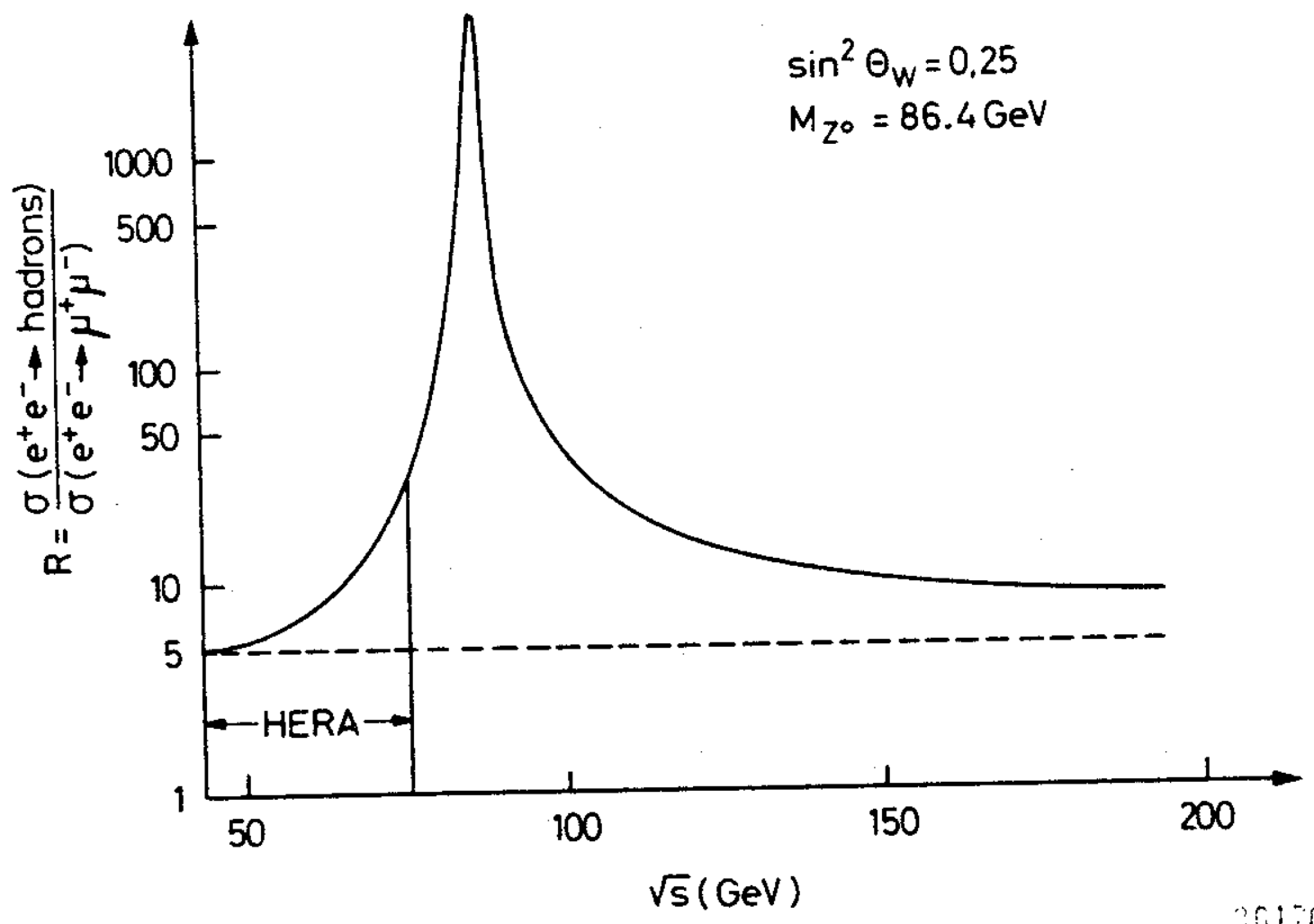

Fig. 16

Annihilation cross section for $\mathrm{e}^{+} \mathrm{e}^{-}+$hadrons evaluated in the standard model with 3 generations and $\sin ^{2} \theta_{w}=0.25$ 


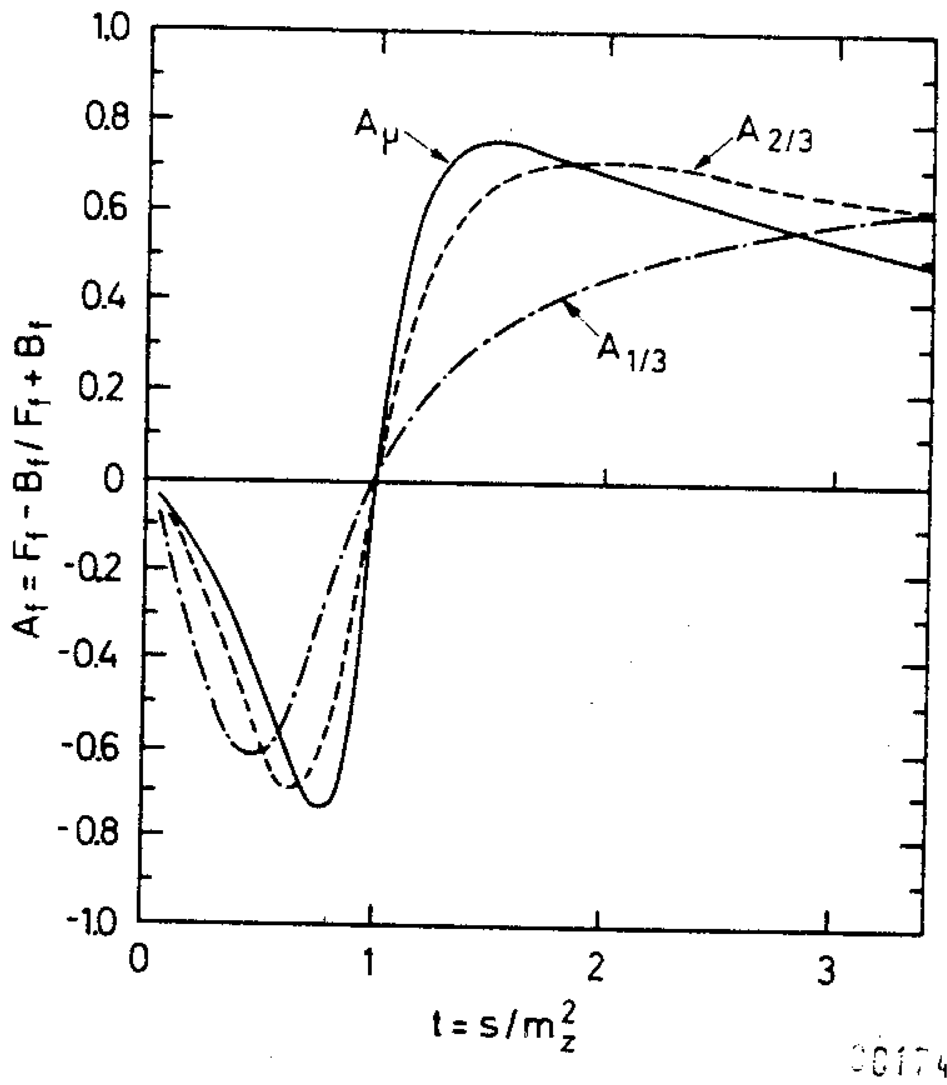

Fig. 17

Forward-Backward asymmetry expected in the standard model for various types of fermions versus $\mathrm{s} / \mathrm{m}_{z}^{2}$ (from ref. 13).

However in order to measure coupling constants one must be able to identify the final state. For example a $c \bar{c}$ final state can presumably be identified by observing leading DD particles in the two back to back jets. However, demanding to observe leading charmed hadrons will lead to a strong reduction in rate. Such measurements will therefore only be possible with a machine optimized for this energy region. This is even more true for heavier quarks.

Toponium, the $1^{-}$state made of $t \bar{t}$ quarks, is generally expected to occur below the $Z^{0}$ peak. The $t \bar{t}$-states and their various decay modes are the ideal laboratory for the study of strong interaction.
For example the decays

$$
\begin{aligned}
& T\left(1^{--}\right) \rightarrow 3 \mathrm{~g}(\mathrm{~g}=\text { gluon }) \quad \text { or } \\
& \mathrm{T}^{\prime}\left(1^{--}\right) \rightarrow \gamma \mathrm{P}_{\mathrm{T}}\left(0^{++}, 2^{++}\right) \rightarrow \gamma g g
\end{aligned}
$$

yield clean gluon jets and can be used to measure the properties of the gluons, - i.e. are they coloured, flavour-neutral spin 1 particles with non-abelian coupings as in QCD.

The decay $\mathrm{T}\left(1^{--}\right) \rightarrow \mathrm{H}^{\mathrm{O}} \mathrm{Y}$ has a large branching ratio, a clean signature and a favourable ratio of signal to background.

Thus Higgs particles with masses rather close to the mass of the $1^{-}$state can be found in these decays.

It might even be possible to determine the number of $v^{\prime} s$ in 
the world by a measurement of $T^{\prime} \rightarrow \pi^{+} \pi^{-} T \rightarrow \pi^{+} \pi^{-} \nu \bar{\nu}$. This measurement however is rather difficult.

It seems rather obvious that the energy region between PETRA and PEP energies and the $Z^{\circ}$ mass is rich and contains information not obtainable from measurements of $z^{0}$ decays. However, to exploit this physics a machine with the luminosity optimized for this energy region is needed. 
HERA

Layout

The layout of HERA on a site ajoining DESY is shown in Fig. 18.

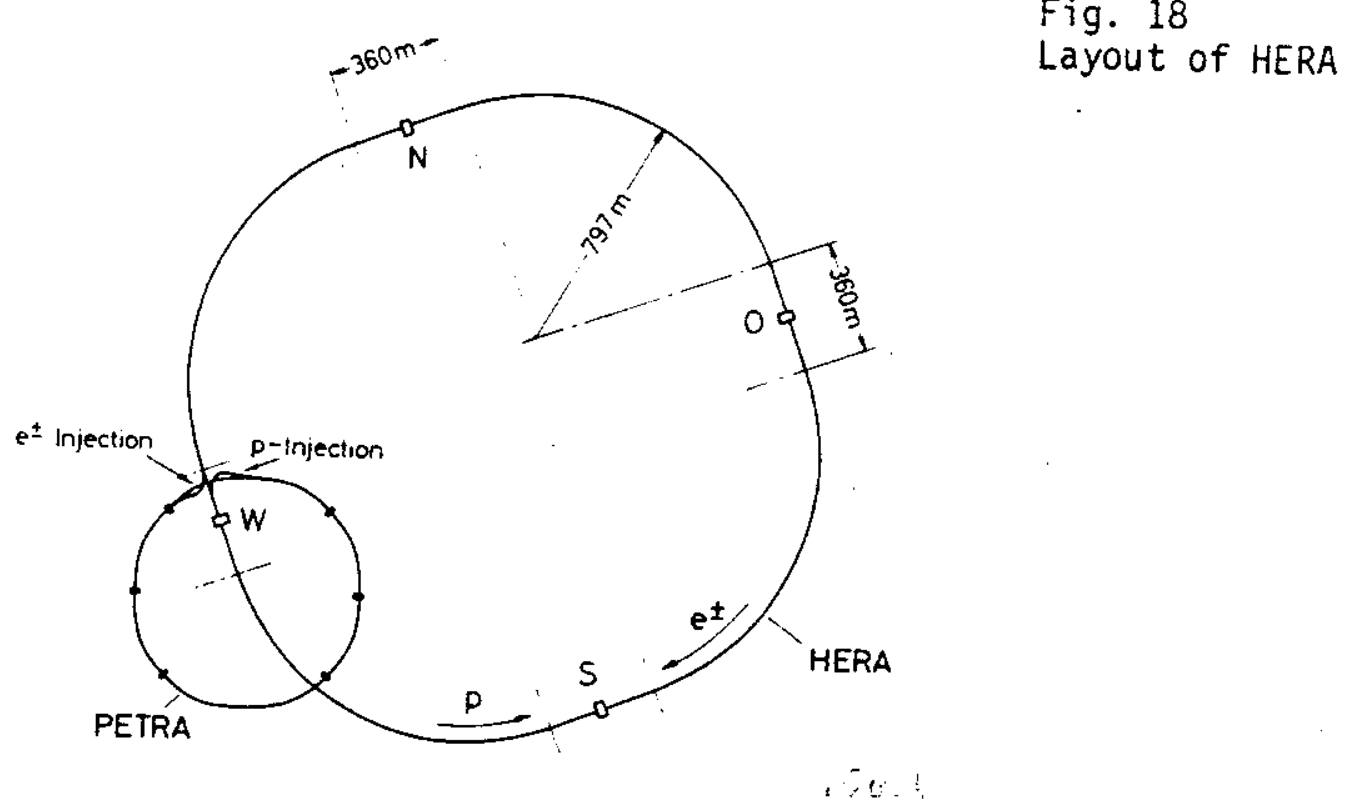

The machine has a fourfold symetry; four $360 \mathrm{~m}$ long straight sections are joined by four arcs with a geometric radius of $797.6 \mathrm{~m}$ yielding a total circumference of $6451.2 \mathrm{~m}$. HERA consists of two rings, one for electrons (positrons), the other for protons and the rings cross the middle of the long straight sections. The machine and the four experimental areas will be burried some $10-20 \mathrm{~m}$ below the surface avoiding any disturbance to the surroundings. The tunnel traverses largely land belonging either to the Federal Government or to the City of Hamburg and it intersects the PETRA ring about $20 \mathrm{~m}$ below the surface. Thus the physical plant can be located on the present DESY site and only short injection paths are needed to connect PETRA and HERA. The site, according to records made available by the Geologisches Landesamt in Hamburg is well suited for tunneling. The tunnel will be drilled using special boring machines equipped with driving shields. These machines, protected by the driving shield, can bore tunnels below the water table without the use of pressurized air. This method has been extensively used in Hamburg and is well adapted to the requirements posed by the HERA tunnel.

A cross section of the tunnel in the arcs with both accelerators and their utilities installed is shown in Fig. 19. To install all this in a tunnel with a diameter of only $3.2 \mathrm{~m}$ amenities like overhead cranes, survey monuments and air conditioning has been dispen-
sed with. 


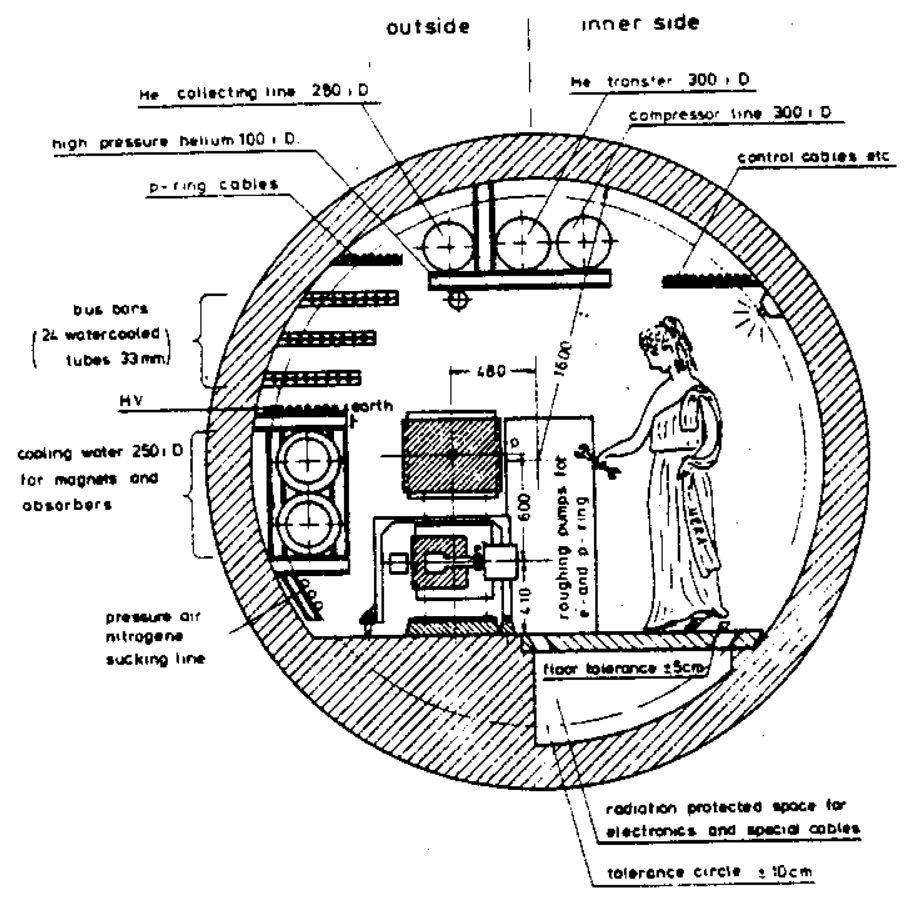

Fig. 19

Cross section

of the tunnel

in the arcs

HERA

cross section in the arc

scale, 25

$\therefore 6:$

The experimental halls are designed to accomodate a small, more specialized experiment, in addition to a large facility type experiment. Also machine components like klystrons, power supplies and compressors must be located in the halls. These requirements have lead to a hall $30 \mathrm{~m}$ long and $50 \mathrm{~m}$ wide with respect to the beam direction. Offices and controll rooms will be located in a multistory structure at the front face of the hall. The halls will be completely covered after construction and the only visible feature will be an access road leading to the elevator shaft which connects the halls with the surface.

Parameters

The general parameters of HERA are listed in table 2 and discussed below.

The maximum electron energy is a compromise between cost and the desire to reach high energies. We have chosen $30 \mathrm{GeV}$ as the nominal upper limit. At this energy the transverse polarization builds up in 19.5 min compared to an expected beam life time of several hours. Note that the polarization time varies with energy as $E^{-5}$. The lower limit on the electron energy is determined by the upper limit on the electron damping time. Electrons of $5 \mathrm{GeV}$ have been stored in PETRA, and if we take this to represent the upper limit on the damping time, electrons down to energies of $10 \mathrm{GeV}$ can be stored in HERA. 
Table 2 - Machine parameters

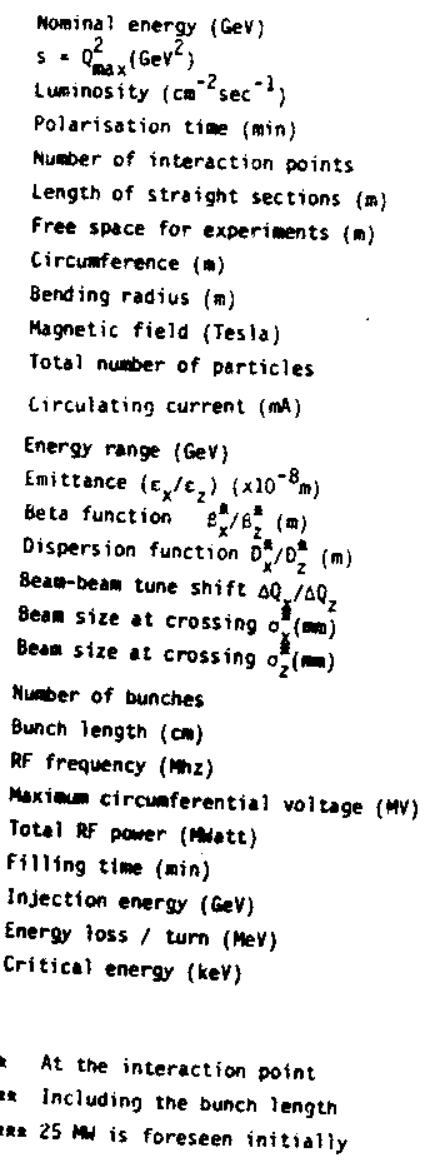

** including the bunch lengen

exz $25 \mathrm{mw}$ is foreseen initialiy

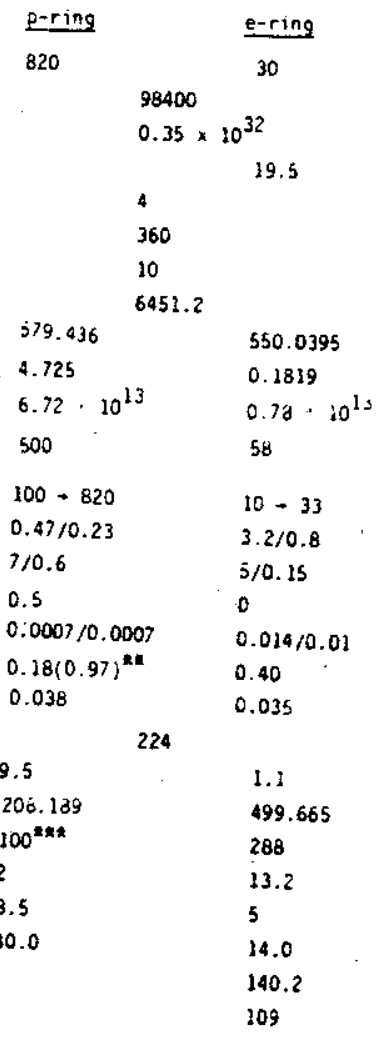

The proton energy for a given geometry is determined by the maximum field strength and the dipole packing fraction in the arcs. Several programs aimed at producing superconducting magnets with niobium-titanum coils have been underway for many years ma inly in the US, but also in Europe. The maximum field of the HERA magnets is chosen to be 4.725 Tesla compared to 4.3 Tesla for the FNAL TEVATRON 14 and 5.0 Tesla, the design value for ISABELLE 15. With a dipole packing fraction of 0.727 in the arcs this yield an maximum proton energy of $820 \mathrm{GeV}$. The lower limit on the proton energy for long term storage is determined by the effect of persistent currents in the superconducting coils. The relative importance of these currents increases with decreasing energy as they cause constant higher multipole fields to disturb the dipole guide field. In particular there is a large sextupole component which leads to a large chromaticity at injection. An estimate of these effects, based on the FNAL magnets, shows that it should nevertheless be possible to inject protons at $40 \mathrm{GeV}$ and to store them down to 
energies of about $100 \mathrm{GeV}$. Note that the lower end of the $Q^{2}$-range available with $200 \mathrm{GeV}$ protons in HERA overlaps with the top Q range explored at FNAL and CERN.

In experiments 16 with a stored bunched beam in the SPS it has been found that the bunch length should be no more than about $30 \%$ of the bucket length. If this condition is violated, then RF-noise will lead to a loss of beam. We have chosen $208 \mathrm{MHz}$ as the RF frequency for the proton beam. At this frequency the bunch will be stable with an RF voltage of $25 \mathrm{MV}$, a voltage which can be provided with high $Q$ cavities at a modest power consumption. A lower frequency would be better but the hardware then becomes cumbersome and expensive.

Several estimates have shown that the cost of an RF system for an electron ring varies little for a choice of frequency between $300 \mathrm{MHz}$ and $700 \mathrm{MHz}$. We have therefore chosen $500 \mathrm{MHz}$, the frequency adopted for the other DESY machines. This choice allows us to exploit fully both the expertise and the hardware available at DESY and makes it attractive to construct the RF system in stages. The final stage employs 192 cavities and a total RF power of $13.2 \mathrm{MW}$, sufficient to reach $35 \mathrm{GeV}$ electron energy with 0 current.

The electron and the proton beam cross in the horizontal plane at an angle of $\pm 5 \mathrm{mrad}$ in the middle of the four long straight sections. A horizontal crossing was chosen since the beam size in the horizontal plane is larger than the beam size in the vertical plane. A system of vertical bending magnets turns the transverse polarization of the electrons in the arcs into a longitudinal polarization in the interaction point. A free space of $\pm 5 \mathrm{~m}$ is foreseen for the experiments. This free space can be made longer at the cost of a loss in luminosity.

The luminosity of an electron-proton colliding ring is given by

$$
L=\frac{f_{0} \cdot n_{b} \cdot N_{e} \cdot N_{p}}{2 \pi\left(\sigma_{x p, e f f}^{2}+\sigma_{x e}^{2}\right)^{1 / 2}\left(\sigma_{z p}^{2}+\sigma_{z e}^{2}\right)^{1 / 2}}
$$

In this formula $f_{0}$ is the revolution frequency, $n_{b}$ the number of bunches in each 0 ring, $N$ and $N_{p}$ the number of electrons and protons per bunch respectigely, $\sigma_{x p, e f f}=\left(\sigma_{x p}^{2}+\left(\sigma_{s p} \cdot \phi\right)^{2}\right)^{1 / 2}$ with $\sigma_{s p}$ denoting the proton bunch length and $\phi$ the crossing angle of $\pm 5 \mathrm{mrad}, \sigma_{x}$ is the width of the electron beam and $\sigma_{z p}$ and $\sigma_{z e}$ the heights $\sigma \theta$ the proton and the electron beam respectivelpy. The $e^{z e}$ beam sizes are all defined in the interaction point and are calculated from the emittances of the two beams. In the case of the proton beam this is determined by the injectors and here we use the standard CERN value of

$$
\varepsilon_{x}=\frac{20 \pi \cdot 10^{-6}}{B \gamma} \mathrm{radm} \text { and } \varepsilon_{z}=\frac{10 \pi \cdot 10^{-6}}{B \gamma} \mathrm{radm}
$$

for the normalized emittances. The emittance of the electron beam is not a constant of motion but determined by the focussing strength 
of the quadrupoles and the synchrotron radiation.

one potential limit to the luminosity is the maximum values of the bean-beam tune shifts. The tune shifts, which must not exceed this maximum, can be expressed as:

$$
\begin{aligned}
& \Delta Q_{z e}=\frac{\beta_{z e} \cdot r_{e} \cdot N_{p}}{2 \pi \gamma_{e}\left(\sigma_{x 0, e f f}+\sigma_{z p}\right) \cdot \sigma_{z p}} \\
& \Delta Q_{z p} \frac{\beta_{z p} \cdot r_{p} \cdot N_{e}}{2 \pi \gamma_{p}\left(\sigma_{x e}+\sigma_{z e}\right) \cdot \sigma_{z e}}
\end{aligned}
$$

Here $\gamma_{e}$ and $\gamma_{p}$ are the electron and the proton energy respectively measured in uhits of the rest energy and $B_{e}$ and $B_{p}$ the values of the amplitude function at the origin. $\Delta Q$ is $P$ quoted per inter-

For a fixed value of the tune shift the number of particles which may be stored and hence the luminosity improves with decreasing values of $\beta$ at the interaction point. The minimum value of $\beta$ is determined by the condition that $\beta_{e}$ must be larger than lengthe $\left(\sigma_{s}\right)$ of $9.5 \mathrm{~cm}$. For the protons $B_{p}$ is $\mathrm{given}$ by the distance between the quadrupoles and the interaction point and the maximum vaiue al lowed for the field in the superconducting coils of the quadrupole. It was chosen to be $60 \mathrm{~cm}$. This results in a maximum value of $B$ elsewhere in the proton ring of about $1000 \mathrm{~m}$.

The luminosity for a given value of the tune shift also increases in proportion to the number of bunches. We have chosen 224 equidistant bunches for each ring correspondjing to a distance of $96 \mathrm{nsec}$ between adjacent bupches. With $3 \cdot 10^{11}$ protons per bunch at $820 \mathrm{GeV}$ and 0.35 . 10 11 electrons per bunch at $30 \mathrm{GeV}$ we obtain a luminosity of $3.5 \cdot 10^{31} \mathrm{~cm}^{-2} \mathrm{sec}^{-1}$. The tune shifts are $7 \cdot 10^{-4}$ and $1.4 \cdot 10^{-2}$ per crossing respectively for protons and

These values for the $Q$ shift are not as high as the limits assumed by proponents of other machines. In fact they are not the primary limits to performance, al though rather close to the tune shift at which beam-beam interaction affects luminosity. HERA's luminosity is limited rather by the currents in the two rings. The electron current is fixed by available RF power while the proton current is 1 imited by single particle instabilities in HERA and its injectors to $3 \cdot 10^{11}$ protons per bunch or a circulating current of 500 mAmp.

The luminosity is plotted in Fig. 20 versus the proton energy for electron energies of $10,20,28$ and $30 \mathrm{GeV}$. The curves are evaluated with the following assumptions: $13.2 \mathrm{MW}$ is the available RF power for the electron beam, $\Delta Q_{p} \leq 0.0025(\Delta Q$ always remains well below 0.03 ) and the proton bunch Tength $\sigma_{\mathrm{s}}=9.5 \mathrm{~cm}$. In the electron ring the phase advance per cell $\Delta \phi{ }^{s}$ is adjusted in discrete steps of $30^{\circ}, 45^{\circ}, 60^{\circ}$, and $90^{\circ}$ to optimize the luminosity. 


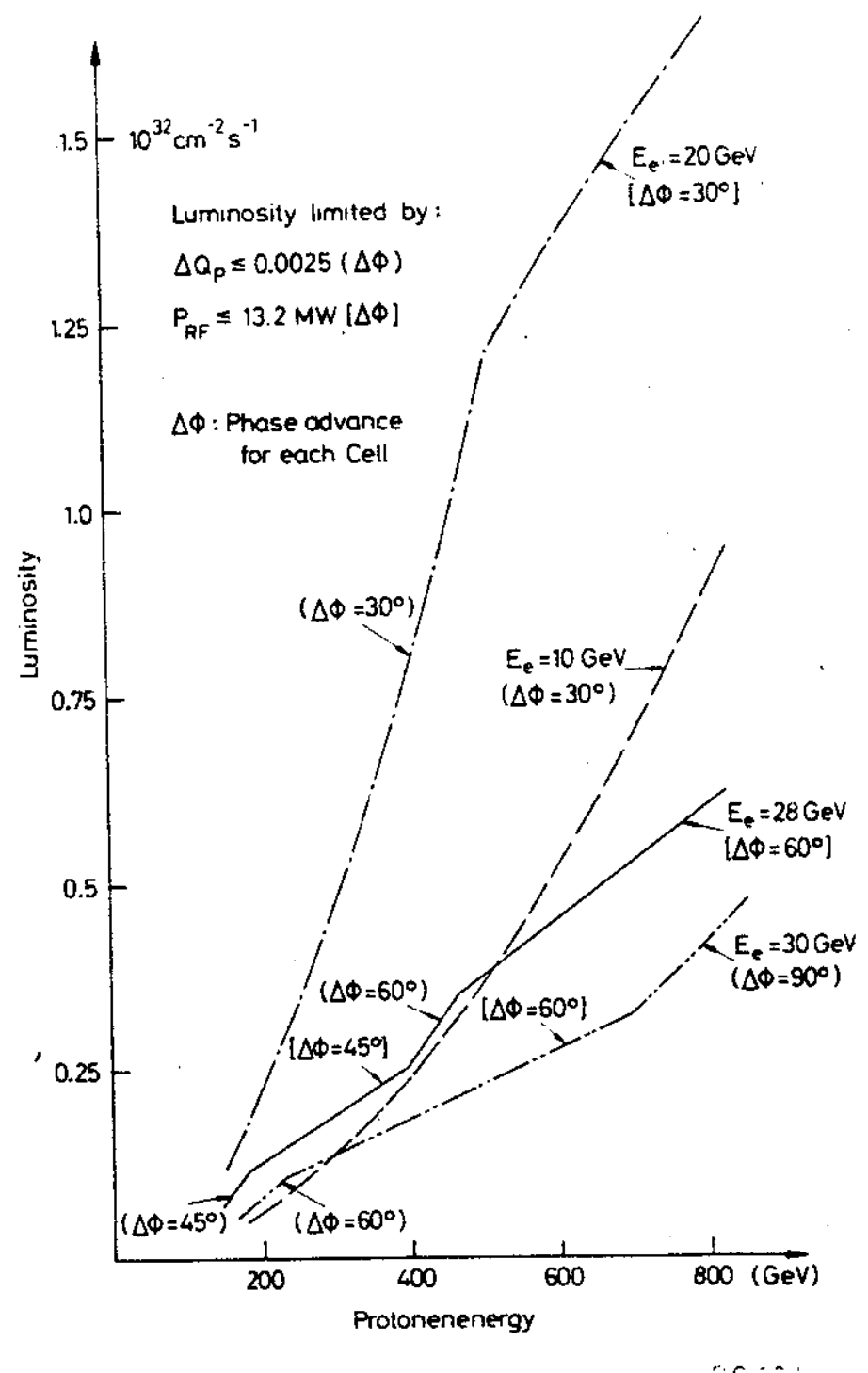

Fig. 20

Luminosity versus

proton energy

$$
2960 \mathrm{i}
$$

At lower proton energies each curve is 1 imited by the tune shift and this is shown by curved parentheses around the $\Delta \phi$ parameter. At higher energies the RF power limits and this is shown by rectangular parentheses.

The injection system is capable of filling the electron ring of HERA with 224 bunches of $14{ }_{1} \mathrm{GeV}$ electrons (positrons) with a maximum intensity of $1.3 \times 10^{11}$ particles per bunch in a time which is short compared to the expected lifetime of the beams. The proposed injection system is based on Linac II, the DESY synchrotron and PETRA and leads to filling times of the order of $10-20 \mathrm{~min}$ for the peak current. 
To a large extent also the proton injection scheme is based on existing accelerators. Protons from a $50 \mathrm{MeV} 1$ inear accelerator are injected into the DESY synchrotron, accelerated to $7.5 \mathrm{GeV}$ and transferred to PETRA where they are accelerated to $40 \mathrm{GeV}$ and injected into HERA. The injection time is of the order of $10 \mathrm{~min}$. To implement the injection scheme DESY must construct a $50 \mathrm{MeV}$ linear accelerator with properties similar to the one used at CERN and equip the DESY synchrotron and PETRA for proton accelera-
tion.

Magnet Layout

Lattice

Both rings have a periodic FODO cell structure consisting of equidistant focusing and defocusing quadrupoles which al ternate in sign. The magnet structure of the standard cells is depicted in

Fig. 21. As much of the interveening space as possibie is filled

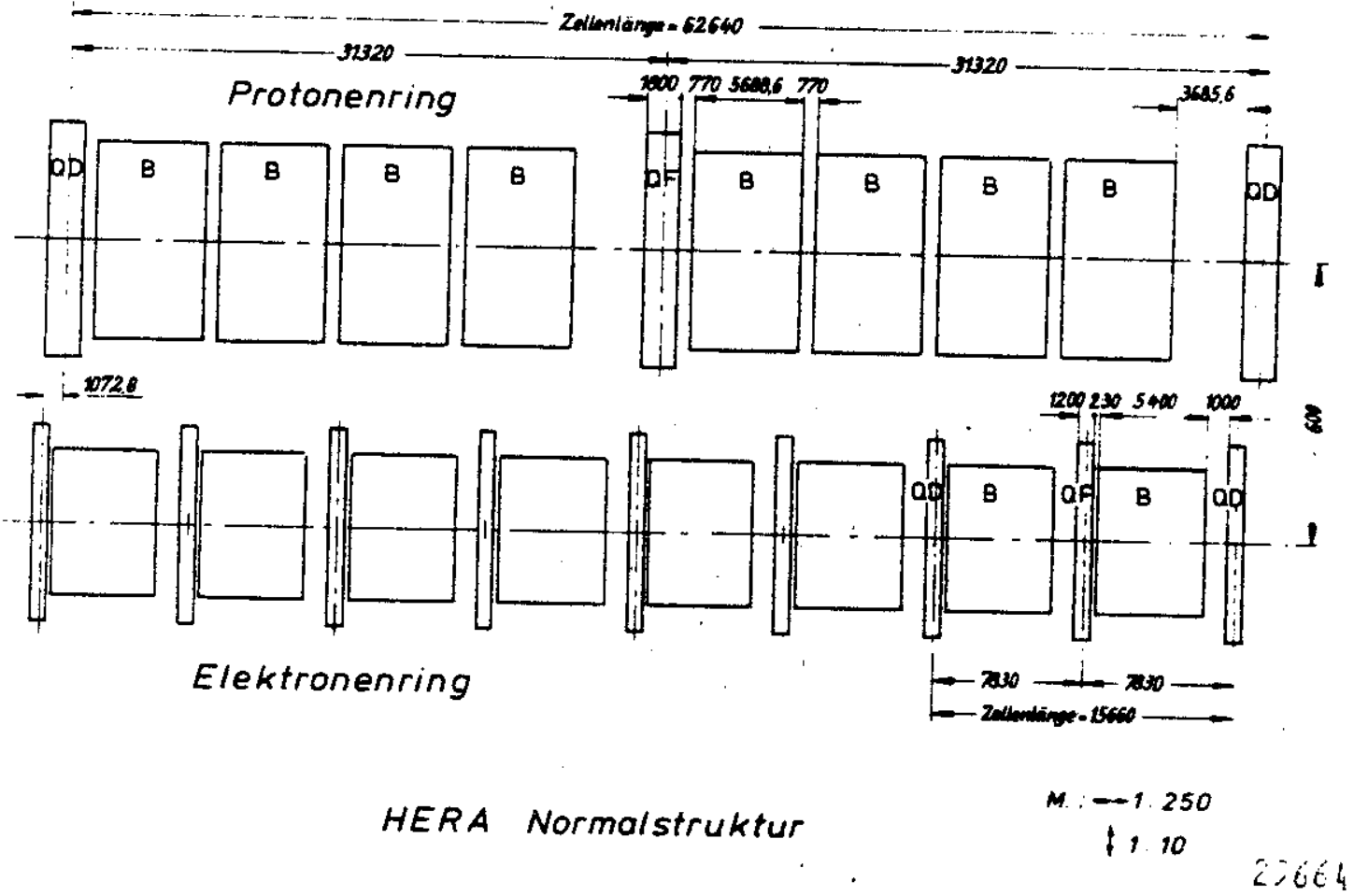

Fig. 21 - Magnet structure in the arcs.

with bending magnets in order to reach the highest proton energy and in case of the electron machine, to minimize synchrotron radiation. A short straight section placed to one side of each quadrupole provides space for sextupole magnets, orbit detecting pickups, correction dipoles and vacuum equipment together with other beam detection equipment and correction windings. 
The length of the proton cell is determined by the need to make the phase advance per cell $90^{\circ}$ while at the same time arriving at a $Q$ value sufficiently high to minimize the effects of nonlinearities. There are 20 proton cells per arc. In contrast there are 80 electron cells per arc reflecting the need to have a dense focusing structure for the electron ring whose design is dominated by synchrotron radiation effects. Such a dense focusing structure leads to a small beam emittance, low synchrotron frequency and low $\mathrm{RF}$ voltage. The number of electron periods is so chosen that with $45^{\circ}$ phase advance per cell the electron beam size matches that of the protons. The lattice can also be run with stronger focusing and $90^{\circ}$ phase advance per cell when the highest electron energy is of interest.

The electron lattice is made of $6405.4 \mathrm{~m}$ long dipole magnets, $6601.2 \mathrm{~m}$ long standard quadrupoles and $56 \quad 1.6 \mathrm{~m}$ long matching quadrupoles and a total of $6040.30 \mathrm{~m}$ long sectupoles.

The proton lattice consists of $6405.69 \mathrm{~m}$ long dipoles and $1641.8 \mathrm{~m}$ long quadrupoles.

Interaction region

Measurements with electrons and positrons in well defined helicity states are needed to untangle electromagnetic and weak effects and to determine the properties of the charged current. Only lefthanded electrons and righthanded positrons have been found to interact via the charged weak current - i.e. all four interaction regions are designed to produce these helicity states.

The insertion is shown in Fig. 22. A side view of the rings is shown in the upper part of the figure. The rings make an $S$ bend antisymetric around the interaction point. The vertical bends in the first part of the straight section cause the polarization vector, which is vertical in the arcs of the machine, to precess until it is antiparallel to the beam in the interaction point. The antisymmetry of the configuration restores the vector to be vertical on reentering the arcs. Each insertion is identical producing identical helicity states in all four interaction points. The precession angle $\theta$ of the spin is related to the electron energy $E$ and the bending angle $\alpha$ by $\theta=2.273 \cdot E \cdot \alpha-i . e$. the precession angle varies with energy. We have chosen $\alpha_{0}=23.04 \mathrm{mrad}$ which precesses the spin of a $30 \mathrm{GeV}$ electron by $90^{\circ}$.

Whereas vertical bends are necessary to produce electrons with well defined helicities, the choice of a horizontal crossing angle or head on collisions is a matter of design. Both possibilities can be realized with the present design - however we have first investigated the geometry shown in the lower part of Fig. 22 with the beams crossing at an angle of $10 \mathrm{mrad}$. The reasons to choose a finite crossing angle can be listed as follows:

1) It is possible to use a continous proton beam. This is advantagous at lower energies where it otherwise might be different to synchronize the beams.

2) The proton quadrupoles can be mounted closer to the interaction point yielding a higher luminosity for the same tune shift. 


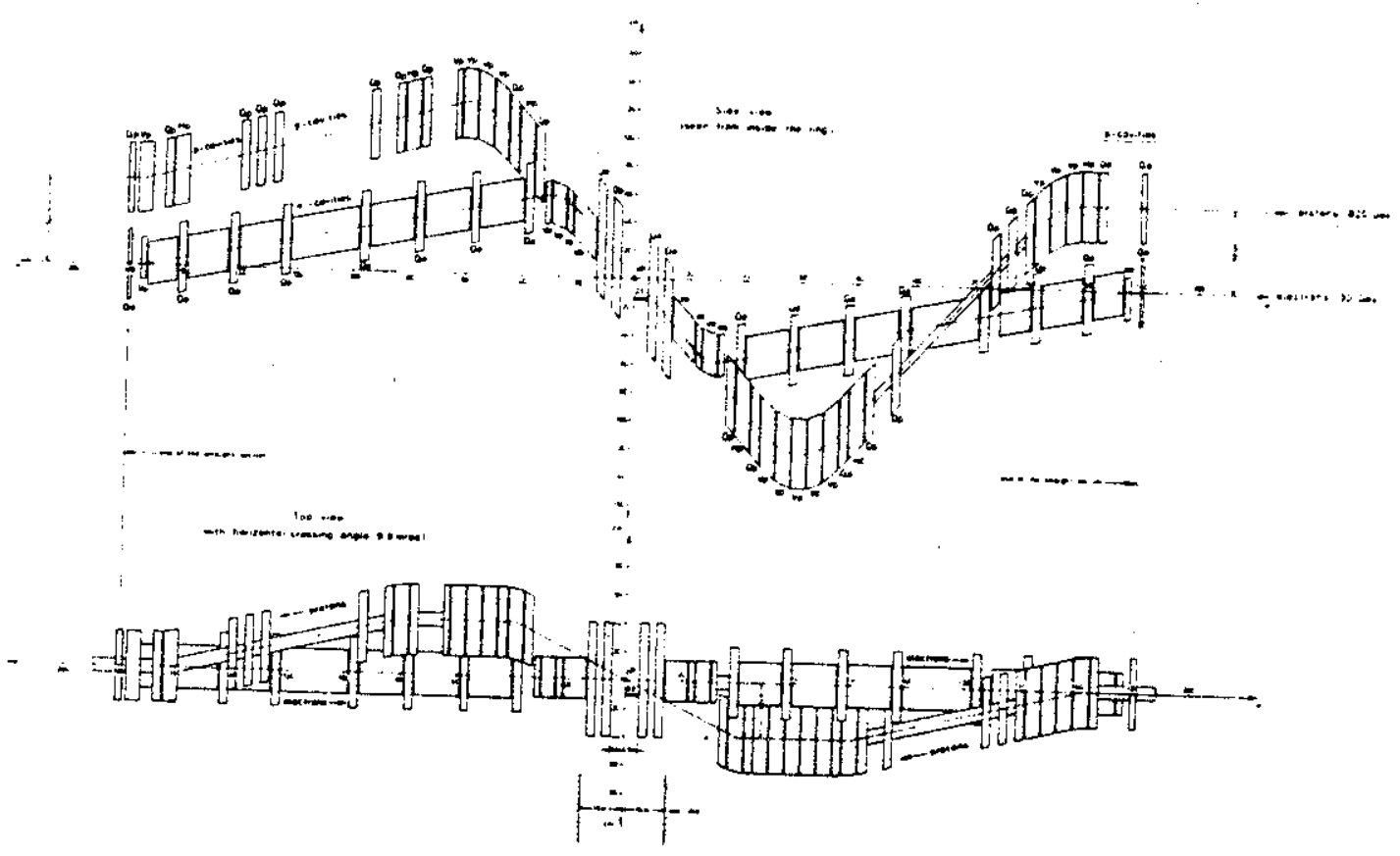

Fig. 22 - The insertion

3) The electron and the proton beams have only a few elements in common. Thus the beam energies can be chosen independently.

4) The synchrotron radiation does not hit the superconducting coils. However the allowable tune shift might be smallerll for a finite crossing angle.

The design shown above minimizes the amount of synchrotron radiation produced adjacent to the interaction point and maximizes the space available to install RF cavities.

Electronring

The electron ring of HERA is similar to the PETRA machine except that the circumference is larger by a factor of 2.8. A1though the PETRA component in principle could be used directly, some changes, based on PETRA experience, are made to simplify the design and to reduce the cost.

The RF system is similar to the one used at PETRA except that the cavity will be designed with seven cells instead of five and a reduced hole size between adjacent cells. These design changes should increase the shunt impedance per meter by $50 \%$ to $\mathrm{R}=$ $18 \mathrm{M} 2 / \mathrm{m}$ and $a$ corresponding reduction in power $W$ for a given voltage $U\left(W=U^{2} / 2 R\right)$. 
The magnets and the vacuumsystem should be considered as a single system. The circulating electron beam is an intense source of synchrotron radiation and instead of trying to contain more than $99 \%$ of the radiation in the beam pipe we propose to use the magnets as absorbers. The beam can be contained both horizontally and vertically in a rather small vacuum chamber. However a small horizontal vacuum chamber makes it difficult to use integrated pumps at the lowest electron energies. We have therefore chosen to use discrete pumps spaced about $2 \mathrm{~m}$ apart. With this arrangement the pumping speed will be independent of energy. A cross section of a dipole magnet with vacuumchamber and a pump is shown in Fig. 23. The vacuumchamber is made of a $4 \mathrm{~mm}$ thick copper pipe.

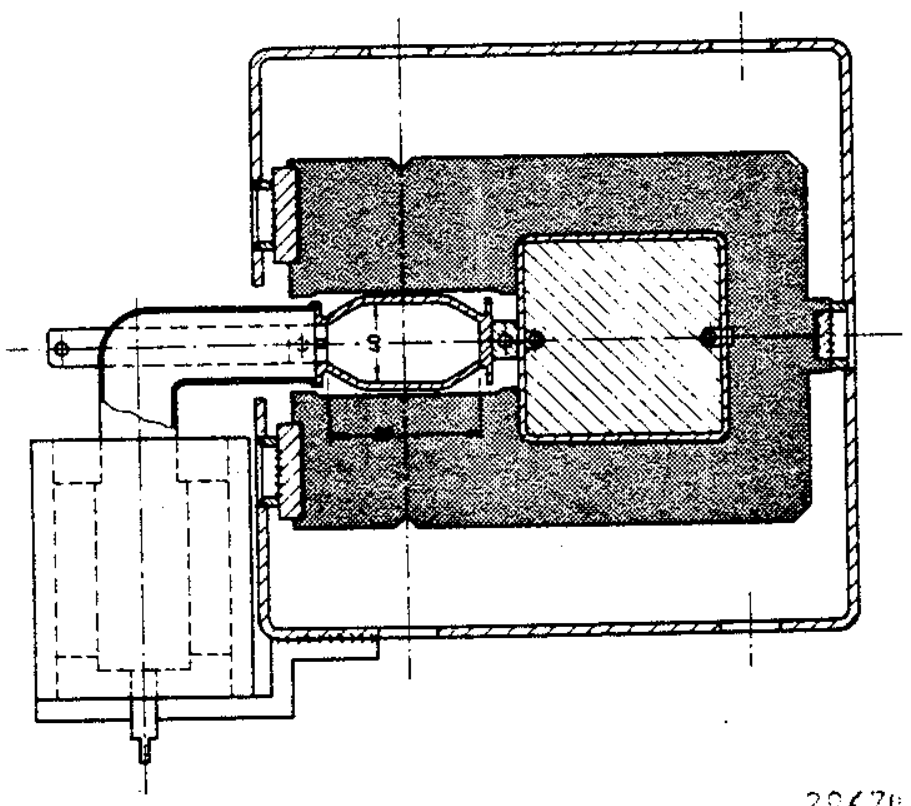

Fig. 23

Cross section of a dipole magnet for the electron ring with vacuumchamber and pump

At $30 \mathrm{GeV}$ about $90 \%$ of the synchrotron intensity is contained in the beam pipe and the remaining $10 \%$ ( $~ 200$ Watt/m) is absorbed in the magnets. During the anticipated ifetime of HERA this is equivalent to some $10^{10}$ rad deposited in the magnet coils. For the simple coil design shown in Fig. 23 it is very easy to insulate the coil using radiation resistant material. This would still be true if, for convenience, we would add a return conductor mounted on the front of the magnet. The coils of the quadrupolmagnets can be shielded by placing lead in front of the coils.

Proton Ring ducting magnets to allow the acceleration and storage of protons at energies up to $820 \mathrm{GeV}$. At this time no superconducting accelerator or storage ring of this size has been completed al though a 

rather similar machine, the FNAL TEVATRON
tion and a $400 \mathrm{GeV}$ proton storage ring complex ISABELLE 15 is being
built at Brookhaven. built at Brookhaven. Altogether 716 dipoles and 236 quadrupoles are needed to guide the protons around the ring and bring them into collisions at the four interaction points.

Field strengths between 4 and 5 Tesla are currentiy produced in magnets which are wound from a niobium titanium superconductor imbedded in a copper matrix. Both the FNAL and the BNL magnets are based on this conductor and we have made the same choice. We have also decided not to cool down the magnet yoke but rather leave it at room temperature as done in the FNAL design. A short cool down time seems to us to be persuasive in view of the large number of magnets to be tested despite strong argements which favour cold iron. Contrary to the FNAL design, however, we decided to use a warm bore, as in the ISABELLE design to avoid any excessive load on the refrigerator system from higher order mode losses. These losses are proportional to the peak current squared and the design peak current in HERA is much above that in the TEVATRON. A warm bore al so allows room temperature corrective and diagnostic equipment to be installed at frequent intervalis in the ring.

A vertical cut through a dipole magnet is shown in Fig. 24.

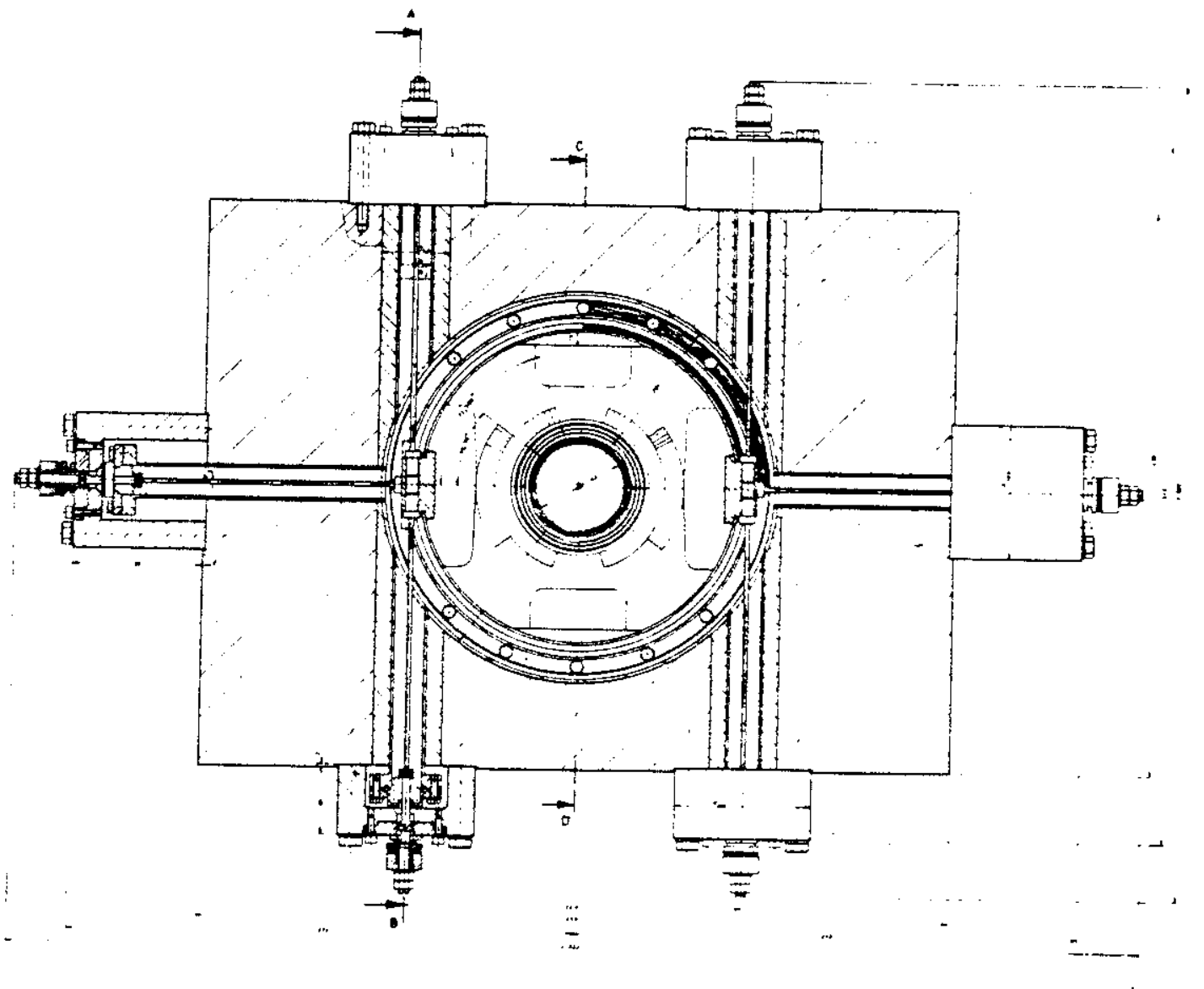

Fig. 24 - Cross section of a superconducting dipole magnet 
The cryostat is mounted inside a $550 \mathrm{~mm}$ wide and $420 \mathrm{~mm}$ high iron yoke using four sets of six tie rods each. The heat loss through the tie rods is small and this system allows us to adjust the coil within the iron following magnetic measurement without warming up the magnet. In the present design the dipole field is approximated by a two shell conductor arrangement fixed by precision stamped stainless steel collars as in the FNAL design.

The coll is immersed in a liquid helium bath at $4 \mathrm{~K}$. The heat shield is maintained at $50 \mathrm{~K}$ by passing cold Helium gas through the outer cryostat.

The parameters of the dipole and quadrupole magnets are listed in table 3 .

Table 3 - Superconducting Magnet Parameters

\begin{tabular}{lll} 
Parameter & Dipole & Quadrupole \\
\hline Magnetic length $(\mathrm{m})$ & 5.686 & 1.80 \\
Induction $(\mathrm{T})$ & 4.725 & - \\
Gradient $(\mathrm{T} / \mathrm{m})$ & - & 74.4 \\
Bore $(\mathrm{cm})$ & 6.3 & 6.3 \\
Current $(\mathrm{A})$ & 6348 & 6348 \\
Critical short sample & & \\
current in cable at & & \\
B $=5.5$ T and the maxi- & & \\
mum tolerable operating & $\mathrm{I}$ & \\
temperature of $t=4.6 \mathrm{~K}$ & ${ }_{\mathrm{cr} .5}(4.6 \mathrm{~K}) \geq 8250 \mathrm{~A}$ \\
Stored energy (kJ) & 914 & 126 \\
Mass (kg) & 8514 & 934
\end{tabular}

An important parameter which is yet to be determined is the required purity of the magnetic field - i.e. the fraction of higher multipole field which might be present in the dipole field without severely restricting the operation of the machine. In the present design we assumed the values given in the FNAL design report 13 .

An overview of the refrigeration system is given in Fig. 25. Rather than one central plant, the refrigeration system is subdivided into 4 units each cooling two octants consisting of $99 \mathrm{di}$ poles and 38 quadrupoles plus one experiment. The total heat load is $16.3 \mathrm{~kW}$ at $4 \mathrm{~K}$ plus $52 \mathrm{~g}$ liquid $\mathrm{He} / \mathrm{s}$. The load can be handeled by 3 of the 4 units. The cryogenic system can thus be maintained and repaired without interrupting operations. The compressors, 10cated on the DESY site, are feeding compressed helium in a warm circuit in the tunnel to the refrigerators which work with expansion turbines and Joule-Thompsen effect. A string of magnets 
is cooled by supercritical helium (i.e. phase one) at a temperature between $3.8 \mathrm{~K}$ and $4.2 \mathrm{~K}$ and a pressure greater than $2.3 \mathrm{bar}$. Because of the limited specific heat of phase one helium, it is recooled by the returning two phase helium between every cell (four bending magnets and one quadrupole). The return stream of low pressure helium is brought back to the compressor through a circuit in the tunnel. To minimize the cross section for this return line the helium from the remote octants is first passed through auxiliary
compressors.

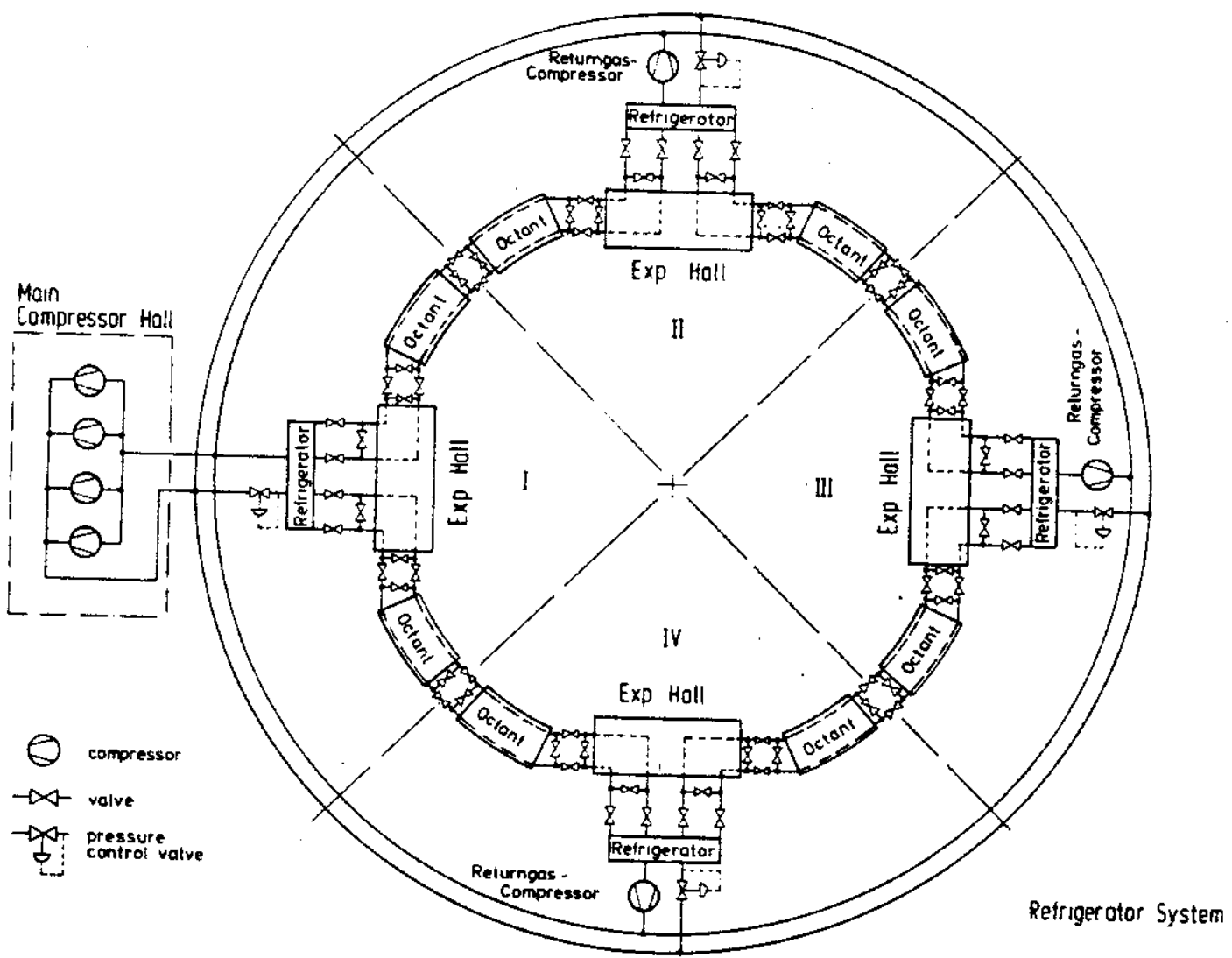

Fig. 25 - Overview of the refrigeration system

Special safety measures are needed in the superconducting system for the case that one or more magnets quench. Without these precautions all the stored energy of the magnet ring might be deposited in a single superconducting magnet which would then be destroyed. As soon as a quench is detected heating strips are fired ensuring that all the magnets in a cell quenches. The magnets will of course be so designed that they can absorb their own stored energy. At the same time the cell is short circuit passing the 
energy of the remaining cells around the quench region. Furthermore the liquid helium in the cell is vented into the return line and the helium flow is passed around the quenched cell to the neighbouring.cells.

DESY is continuing the machine studies with the aim of submitting a detailed proposal in 1981. In parallel a vigorous effort to design and build prototype superconducting magnets is underway. This work is done in collaboration with both Saclay and industrie and is expected to yield the first dipole and quadrupole prototypes in the middle of 1982 . The preparation for the civil engineering work is progressing well and the necessary construction permits might be obtained towards the end of 1981 such that construction would start early 1982. Once the project has been approved the construction work will be completed in four years such that the electron ring could be operating in the fifth year and the proton ring in the seventh year of construction. The two years between the completion of the electron ring and the turn on of the proton ring will be used to install the proton magnets and to commission the electron ring - i.e. learn how to operate a high current multibunch electron machine and how to produce polarized electrons. However, we also hope that some time will be available for $e^{+} e^{-}$ physics using the central part of the ep-detectors.

The HERA program is very ambitious, it is based on new technologies and it tries to collide electrons and protons which has never been done before. However, the scientific rewards should be great and HERA wOuld ensure DESY of an exciting future. 
References

1. Report of the Electron Proton Working Group of ECFA Study of the Proton-Electron Storage Ring Project HERA.

This study was organized by U.Amaldi with the following convenors: Theory: G.Altarelli, J.Ellis, G.Kramer and C.H.Llewellyn-Smith

Machine: B.H.Wiik and E.J.N.Wilson -

Experiments: D.Dalpiaz, W.Hoogland, H.E.Montgomery, D.H.Perkins, P.Söding, K. Tittel and R. Turlay

Superconducting magnets: G.Horlitz

2. H.G. Hereward, K.Johnsen, A.Schock and C.J.Zilverschoon Proc. 3rd International Conference on High-Energy Accelerators,
Brookhaven, 1971 p. 265

L.Goldzaht and E.G.Michael is, CERN 66-12 (1966)

3. C.Pellegrini, J.Rees, B.Richter, M.Schwartz, D.Möhl and A.Sessler Proc. 8th International Conf.on High-Energy Accelerators

4. H.Gerke, H.Wiedemann, B.H.Wiik and G.Wolf DESY H-72/22 (1972)

M. Tigner, DESY Techn. Notiz 2/73

5. E.Dasskowski, R.D. Kohaupt, K.Steffen, G.A.Voss DESY 78-02 (1978)

6. R. Chasman and G.A.Voss, IEEE Trans. Nucleus Science NS-20, No. 3 (1973) 777

EPIC Machine Design Study Group, 9th International Conf. on

High Energy Accelerators, Stanford, 1974, p. 548

T.L.Collins, D.A.Edwards, J.Ingebretsen, D.E. Johnsen,

S.Ohnuma, A.G. Ruggiero and L.C. Teng

IEEE Trans. Nucleus Sci. NS-22, No. 3 (1971) 1411

G.E.Fisher, D.Bleckschmidt, A.Hofman, H. Hoffmann and B.W.Montague

B.H.Wiik pg 220 in CERN 76/12 (1976)

CHEEP - An e p facility in the SPS

J.El] is, B.H.Wiik and K.Hübner (editors)

TRISTAN PROJECT

T.Nishikawa, in proceedings of the 9 th International Conference on High Energy Accelerators, SLAC (1974) p. 584

for an update see

Y. Kimura et a., KEK report

7. C.H.Llewellyn-Smith and B.H.Wiik - DESY 77/38 (1977)

8. PETRA, updated version of the PETRA Proposal, DESY Hamburg 1976
9. S.L.Glashow, Nucl.Phys. 22 (1961), 579

S.Weinberg, Phys.Rev.Lett. 19,(1967), 1267

A.Salam, Proc. 8th National Symposium, Stockholm,

Almquist and Wiksells, Stockholm 1968 p. 363

10. A.J.Buras and K.J.F.Gaemers, Nucl.Phys. B 132 (1978), 249

11. For reviews see: H.D.Politzer, Phys.Rep. 146 (1974), 129

M.Marciano, H.Pagels, Phys.Rep. 366 (1978), 137

U.Ellis, CERN-preprint TH 2744 to appear in the Proceedings of the 1979 Int. Symposium on Lepton and Photon Interactions, 
12. For a review and references, see G.Barbiellini et al., DESY 79/67 (1979)

13. K.Winter, LEP Summer Study / 1-4 (January 1979)

14. TEVATRON - Superconducting Accelerator Design Report (1979) Fermi National Accelerator Laboratory

15. ISABELLE - a $400 \times 400 \mathrm{GeV}$ Proton-Proton Coll iding Beam Facility - Brookhaven National Laboratory, BNL 50718 (1978)

16. D.Boussard et al., IEEE Transactions on Nuclear Science, NS-26, No. 3 (1979)

17. A.Piwinski - DESY $77 / 18$. 
